\title{
A View to the Future: Ultraviolet Studies of the Solar System
}

\author{
Noah Brosch · John Davies • Michel C. Festou ${ }^{\dagger}$. \\ Jean-Claude Gérard
}

Received: 9 August 2005 / Accepted: 21 November 2005

(C) Springer Science + Business Media B.V. 2006

\begin{abstract}
We discuss the status of ultraviolet knowledge of Solar System objects. We begin with a short historical survey, followed by a review of knowledge gathered so far and of existing observational assets. The survey indicates that UV observations, along with data collected in other spectral bands, are necessary and in some cases essential to understand the nature of our neighbors in the Solar System. By extension, similar observations are needed to explore the nature of extrasolar planets, to support or reject astro-biology arguments, and to compose and test scenarios for the formation and evolution of planetary systems.

We propose a set of observations, describing first the necessary instrumental capabilitites to collect these and outlining what would be the expected scientific return. We identify two immediate programmatic requirements: the establishment of
\end{abstract}

\begin{tabular}{l}
\hline Deceased 11 May 2005 \\
\hline Dedication: Michel Festou, our co-author and a very important \\
contributor to this paper, passed away while this paper was being \\
completed. We dedicate it to his memory
\end{tabular}

\section{N. Brosch}

The School of Physics and Astronomy, Beverly and Raymond Sackler Faculty of Exact Sciences, Tel Aviv University, Tel Aviv 69978, Israel

J. Davies

UKATC, Royal Observatory, Blackford Hill, Edinburgh EH9 3HJ, UK

\section{C. Festou}

Observatoire Midi-Pyrénées 14, avenue E. Belin 31400 Toulouse, France

J.-C. Gérard

Laboratoire de Physique Atmospherique ét Planetaire, Universite de Liege, allee du 7 aout,

4000 Liege, 94720 Belgium a mineralogic database in the ultraviolet for the characterization of planetary, ring, satellite, and minor planet surfaces, and the development and deployment of small orbital solar radiation monitors. The first would extend the methods of characterizing surfaces of atmosphere-less bodies by adding the UV segment. The latter are needed to establish a baseline against which contemporaneous UV observations of Solar System objects must be compared.

We identify two types of UV missions, one appropriate for a two-meter-class telescope using almost off-the-shelf technology that could be launched in the next few years, and another for a much larger (5-20 meter class) instrument that would provide the logical follow-up after a decade of utilizing the smaller facility.

Keywords Planets · Comets · Solar system · Ultraviolet

\section{Introduction}

The UV window extends from 20 to $400 \mathrm{~nm}$. It covers the spectral domain where atoms have most of their resonance lines and where simple ions and molecules have their fluorescence transitions. This is also the region where most molecules and atoms are photodissociated and photoionized. Below $100 \mathrm{~nm}$ is the spectral segment where most rare gases have their resonance lines. Solar system objects can be observed in this spectral region either because they harbour hot or violent environments or because the solar light that is being absorbed and then scattered by them contains thousands of lines below $200 \mathrm{~nm}$.

One of the basic questions in modern astrophysics is how planets "work," how planetary systems originated, and how life emerged on Earth. By studying our Solar System we are linking 'local' studies to the issue of the existence of 
Earth-like extrasolar planets and the conditions expected on their surfaces. The discovery of biomarkers on Earth analogs is the essence of the search for extra-terrestrial life addresses the question "are we alone in the Universe?" These are the basic questions being asked by NASA and ESA: "How did our Earth and our Solar System evolve, where are we in the Universe, where are we going, and where did life come from?"

Planets and other minor bodies represent the end stage of the Solar System's formation and their present state is the result of numerous accretion, coalescence, and evolution processes. The various components are inter-related: planets were formed through planetesimal accretion, evidence of which remains in the form of asteroids and other bodies such as Trans-Neptunian Objects (TNOs). Some comets are fragments of TNOs; others come from the Oort cloud, both in very cold and protective environments of the Solar System. Thus the minor bodies of the Solar System have retained information that allows us to study the primordial cloud that formed our Solar System some 4.5 Gyrs ago. The traditional rocky asteroids are intermediate objects, between planetesimals and fully-grown planets, and thus contain information on the stage of nebular matter condensation prior to the planet accretion. The collisional history of the Solar System can be investigated by studying the internal structures and size distributions of asteroids, TNOs and Near Earth Objects (NEOs). Finally, the interplanetary dust is mostly the result of the "grinding down" of Main Belt asteroids, probably TNO debris, and ejecta from cometary nuclei.

While many objectives of solar system research can be achieved by optical and near-IR (nIR) imaging, surface mineralogic characterization requires a wide spectral range including the UV. Observations of planetary aurorae from the Earth are impossible, owing to the brightness of the sunlit planetary disks and the lack of contrast at visible and nIR wavelengths. Planetary missions with UV capabilities (such as the Mercury Atmospheric and Surface Composition Spectrometer (MASCS) on the Mercury probe MESSENGER, and the ALICE instrument on the Pluto/Kuiper Belt New Horizons mission) are rare and far apart; so it is necessary to consider Solar System studies in the context of general-purpose astronomical payloads. Even though a UV space telescope might not be fully dedicated to planetary astronomy, a complement of valuable targets exists and Solar System observations can be made without compromising the astrophysical goals of such a mission.

Planetary studies require synoptic observations over periods of time ranging from a single revolution (hours to days), to the length of a comet passage through the inner planetary region and the seasons of telluric planets (months), to orbital revolutions about the Sun and to seasons on giant planets and their satellites (months to years). Since some planetary phenomena, such as aurorae, are directly linked to the solar activity, studies should span one or more 11-year solar cycles. The variability of Solar System objects, sometimes on short time scales, underlines the need for long-term studes to separate intrinsic from evolution-driven properties of planetary bodies and comets.

For a given aperture size, UV astronomy from space can achieve much higher spatial resolution than from the ground because of the absence of the smearing effect of the Earth's atmosphere ("seeing") and because of the smaller diffraction limit of UV telescopes. Present-day 8-m and larger groundbased optical telescopes, equipped with adaptive optics (AO) and with fields of view restricted to the "coplanarity patch," offer the same angular resolution as a two-meter space UV telescope but are restricted to the optical-near IR bands and are hampered by the natural sky brightness. The sky background at UV wavelengths is darker by about five magnitudes than at the best ground-based observatories (O'Connell 1987) and allows observations of very faint objects, in particular those with extended very low surface brightness features.

Why argue now for UV astronomy? Few instruments that allow UV planetary observations are presently available and none will be available in the near future as the few existing missions reach their design and even extended lifetimes. The heritage of past missions, and the expertise of scientists in designing, performing, and analyzing UV planetary observations are dwindling as individuals reach the end of their active careers. New scientists refrain from entering a field devoid of a promise to access cutting-edge research instrumentation. There is need to continue synoptic observations of variable sources even after specific planetary probes complete their missions, and it is necessary to maintain the know-how of observing and working with specific data sets.

\section{Review of achievements}

The study of the UV emission from Solar System bodies, which started as an exploratory task in the middle of the last century, proved useful for the understanding of planetary atmospheres and of plasma phenomena. Results obtained about distant bodies were applied to the understanding of our Earth. Spectroscopy of comets in the UV revealed the presence of new compounds and clarified the mechanisms accounting for their presence.

Key missions of solar system UV exploration

Rocket flights of (very) short durations provided the first exploratory data and flights of that nature still remain useful for developmental and testing purposes. As late as 2004 a Black Brant rocket lofted a telescope and spectrometer to record the UV emission from Mercury. Modern payloads 
Table 1 Past space missions that performed UV observations of Solar System bodies

\begin{tabular}{|c|c|c|c|c|c|c|}
\hline Dates & Mission & Agency & Instrument & Resolution & Range (nm) & Comment \\
\hline 1968 & OAO-2 & NASA & $\begin{array}{l}\text { Photometer }+ \\
\text { spectrometer }\end{array}$ & $\sim 100$ & $100-400$ & $\begin{array}{l}\text { H coma of Comet } \\
\text { Tago-Sato-Kosaka }\end{array}$ \\
\hline 1970 & OGO-5 & NASA & $\begin{array}{l}\text { Lyman alpha } \\
\text { photometer }\end{array}$ & Low & 121.6 & $\begin{array}{l}\text { Interplanetary H. Comets } \\
\text { Bennett and P/Encke }\end{array}$ \\
\hline 1972 & TD-1A & ESRO & $\begin{array}{l}\text { S-59+ S2/68 } \\
\text { Spectrometers }\end{array}$ & $\sim 70$ & $133-280$ & UV sky survey \\
\hline 1975 & ASTP & NASA & EUV Telescope & Imager & $5-150$ & Manned space flight \\
\hline 1977-1989 & Voyager $1 / 2$ & NASA & UV Spectrometer & $\sim 40$ & $40-180$ & Planetary Mission \\
\hline 1972-1981 & OAO-3 Copernicus & NASA & UV Telescope & $\sim 200$ & $75-300$ & Giant Planets and Comets \\
\hline 1983 & Astron & USSR/CNES & Spectrometer & $\sim 200$ & $150-350$ & P/Halley \\
\hline $1993 \& 96$ & ASTRO-SPAS & NASA/DARA & ORPHEUS & ５000 Spectrometer & 39-91 & Shuttle Free Flyer \\
\hline $1993 \& 96$ & ASTRO-SPAS & NASA/DARA & IMAPS & $\sim 200,000$ Spectrometer & $95-115$ & Shuttle Free Flyer \\
\hline $1990 \& 95$ & ASTRO 1/2 & NASA & HUT & $\sim 100$ & $45-185$ & Venus, Mars-Polarimetry \\
\hline $1990 \& 95$ & ASTRO $1 / 2$ & NASA & WUPPE & $\sim 100$ & $140-320$ & Moon, Mars, Io, Halley \\
\hline $1990 \& 95$ & ASTRO $1 / 2$ & NASA & UIT & Imager & $120-300$ & \\
\hline 1978-96 & IUE & NASA/ESA & UV Spectrometer & $\sim 50$ and $\sim 5000$ & $115-320$ & $\begin{array}{l}\text { Giant Planets, Aurora, 50+ } \\
\text { Comets }\end{array}$ \\
\hline $1992-2000$ & EUVE & NASA & $\begin{array}{l}\text { EUV Spectrometer } \\
\text { and Imager }\end{array}$ & $\sim 400$ & $8-75$ & $\begin{array}{l}\text { Sky Survey \& follow-up, } \\
\text { comets, Venus, Jupiter }\end{array}$ \\
\hline 1989-2003 & HST & NASA/ESA & WFPC, GHRS & Imaging, 2000-80000 & $>115$ & Superb images \\
\hline 1998-2004 & HST & NASA/ESA & STIS & $100-100000$ & $115-310$ & All planetary objects \\
\hline $2000-$ & FUSE & NASA & $\begin{array}{l}\text { High Resolution } \\
\text { Spectrometer }\end{array}$ & $\sim 27,000$ & $90-120$ & $\mathrm{H}_{2}$ on Mars, comets \\
\hline
\end{tabular}

Not included in this list are several small experiments carried on various missions such as Cosmos 51, 215, 262, Apollo 16, ANS, D2B-Aura, Skylab and Soyuz/Salyut, as well as short-duration rocket flights.

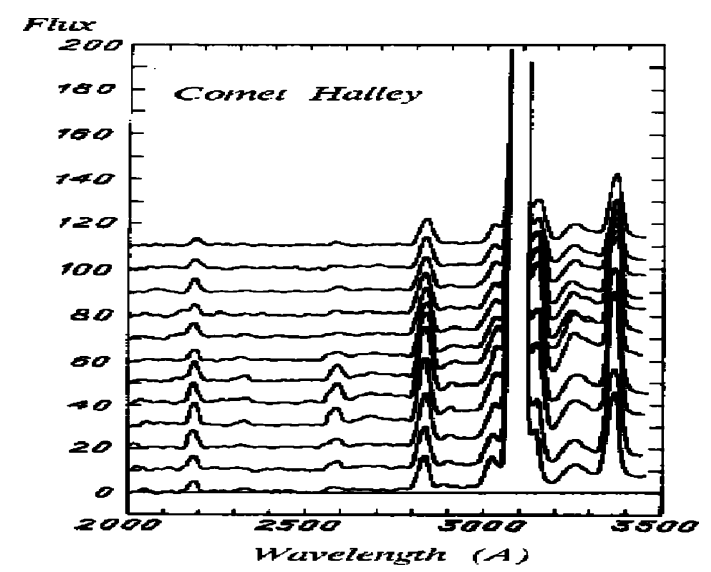

Fig. 1 Time series observation of the UV spectrum of P/Halley obtained by the ASTRON orbital observatory (see Table 1) showing the strong $\mathrm{OH}$ feature at $308 \mathrm{~nm}$.

[http://www.crao.crimea.ua/astron/astron.html]

can achieve more than a simple exploration but still lack the temporal coverage characteristics of Solar System object phenomena.

The Voyager spectrometers were non-imaging, with mechanical collimators defining their fields of view. Their spectral coverage was from 40 to $180 \mathrm{~nm}$, the throughput was rather low and no spatial information was available. Even so, their results regarding the properties of giant planet atmospheres, obtained by occultation techniques or in direct "airglow" mode, were unique because of the proximity of the instruments to their targets.

NASA's Copernicus satellite (Orbiting Astronomical Observatory-3, launched in 1972 and operated till February 1981) performed Far-UV (FUV) investigations and allowed the detection of important atomic and molecular species of the local interstellar medium, among which molecular hydrogen and deuterium. OAO-3 offered a unique way to study the upper atmospheres of planets and key components of cometary atmospheres.

Copernicus was followed by ORFEUS (Orbiting and Retrievable Far and Extreme Ultraviolet Spectrometer) and by HUT (Hopkins Ultraviolet Telescope), both Shuttlelaunched and retrievable payloads. HUT flew twice on the ASTRO platform for missions of order 12 days, along with WUPPE (Wisconsin Ultraviolet Photo-Polarimeter Experiment), the only instrument that has provided polarization measurements in the UV. WUPPE was used to study the Moon, Mars, Io, and comet Halley. HUT observed Jupiter, Venus and Mars. IMAPS (Interstellar Medium Absorption Profile Spectrometer) operated, as ORFEUS did, on board the AstroSPAS space shuttle-borne platform (1993).

Most missions mentioned above were limited in duration or in the observing time allocated for Solar System studies. 
Their role was, by necessity, mainly exploratory. The exciting results indicated the need for missions of much longer duration.

The spectroscopic results obtained by the International Ultraviolet Explorer (IUE) spacecraft with its $45-\mathrm{cm}$ telescope surpass by far those obtained by any other spacecraft, perhaps with the exception of the Hubble Space Telescope (HST). IUE was launched in January 1978 and its three-year mission was extended year after year until it was deliberately terminated in September 1996. These mission extensions provided much of the most valuable science return. IUE performed comparative studies of auroral activity at Jupiter, Saturn, and Uranus and demonstrated the impact of solar wind variations on the brightness of the Jovian aurora. The amount of hydrocarbon absorption in auroral spectra was used to determine the FUV color ratio, from which the energy of the primary auroral electrons could be inferred. The spectra revealed the existence of new sulphur-bearing compounds in cometary spectra $\left(\mathrm{S}_{2}\right.$ in comet IRAS-Araki-Alcock; $\mathrm{CS}_{2}$ in numerous comets) and investigated the abundance of water and carbon compounds such as $\mathrm{CO}$ and $\mathrm{CO}_{2}$, providing a database on gas production rates in over 50 comets that is only surpassed in size by $\mathrm{OH} 18 \mathrm{~cm}$ radio surveys and by the ground-based survey of the UMD-Lowell group (A'Hearn 1995).

The field of Extreme UV (EUV) observations was covered by the Extreme Ultra Violet Explorer (EUVE) satellite, launched in 1992 and operated until 2000. EUVE detected emission from comets resulting from charge-exchange reactions with highly charged Solar wind particles, observed the dayglow of the atmosphere of Venus, showed the existence of EUV emission from the Full Moon that indicated differences on the local albedo, and detected helium emission from the atmosphere of Jupiter following the impact of $\mathrm{km}$-sized disruption fragments from D/Shoemaker-Levy-9 (SL9).

The workhorse of solar system studies in the field of highresolution imaging, or observations in spectral domains not visible from the Earth's atmosphere, has been the Hubble Space Telescope (HST), a 2.4-meter telescope for the UVto-NIR domain launched in 1990 . The HST has produced the best imaging database of other celestial bodies obtained from the Earth's vicinity. The HST results range wide, from atmospheric studies of the giant and the terrestrial-like planets, to imaging the dynamics of Jupiter's and Saturn's aurora down to 10 -s variations and monitoring the energy of the impinging auroral electrons in regions connected to different magnetospheric plasma sources, to single-pixel imaging of Sedna and the coarse mapping of the surfaces of Pluto, 1 Ceres and 4 Vesta. HST tracked the disintegration of comets (SL9 and C/1999 S4 (LINEAR)) and found ozone on Ganymede and molecular oxygen in the atmosphere of Europa. With the demise in 2004 of the Space Telescope Imaging Spectrometer (STIS) and with a refurbishing mission that could repair or replace STIS doubtful, the lack of a general-purpose UV spectroscopic facility is becoming acute.

The Far Ultraviolet Spectroscopic Explorer (FUSE) satellite was launched in 2000; it covers the spectral range from 90.5 to $119.5 \mathrm{~nm}$ with reasonably high resolution $(R=$ $24,000-30,000)$ and is still operating when these lines are written. FUSE has a 352 by $387 \mathrm{~mm}^{2}$ aperture and a PSF of 1.5 arcsec. The FUSE spectral range was chosen to contain the most important interstellar and hot environment lines of deuterium, $\mathrm{H}_{2}$, and lines arising from high level ionization states of the most abundant atoms in the universe $(\mathrm{O}, \mathrm{C}$, $\mathrm{N}, \ldots)$. FUSE has a relatively low sensitivity, with effective areas of $20 \mathrm{~cm}^{2}$ at $90 \mathrm{~nm}$ and $80 \mathrm{~cm}^{2}$ at $120 \mathrm{~nm}$, although this is 10,000 times the sensitivity of Copernicus and the resolution is much better than any of the space FUV instrumentation built before. Among the achievements of FUSE in the field of Solar system studies we count the discovery of $\mathrm{H}_{2}$ on Mars (Krasnopolsky and Feldman 2001), presumably the result of photo-dissociation of water and subsequent molecular formation at mid-altitudes of the Martian atmosphere, the measurement of the D-to-H ratio from which the existence of an old global ocean on Mars can be inferred, the detection of numerous new lines in comet spectra and an unprecedented coverage of the auroral phenomena in the giant planets. The measured intensity distribution amongst $\mathrm{H}_{2}$ lines affected by self-absorption was used to infer the pressure level of the Jovian aurora, which was shown to be quite different from Saturn's case.

The FUSE spectra of comet C/2001 A2 LINEAR provided the first high resolution EUV spectrum of a comet. In addition to the $\mathrm{H}_{2}$ lines, many new lines have been discovered. Quite a few of these features are still unidentified and require more work to be understood, and some of them likely are due to electron impact excitation.

Key results from past UV astronomy missions

\section{Planetary atmospheres and magnetospheres}

Results from comparative studies of planetary atmospheres indicate that the Solar System family can be separated into four groups:

1. Bodies with Nitrogen dominated atmospheres (Earth, Titan, Triton, Pluto)

2. Bodies with carbon dioxide dominated atmospheres (Venus and Mars)

3. Hydrogen \& Helium dominated giant planets (Jupiter, Saturn, Uranus, and Neptune)

4. Bodies with thin atmospheres, separable further into three subgroups:

a. Rocky surfaces (Mercury, the Moon)

b. Volcanic bodies (Io)

c. Icy surfaces (Europa, Ganymede, Callisto, Charon) 
Fig. 2 The EUV long wave spectrum of comet Hyakutake barely shows the emission lines originating from

charge-exchange reactions with solar wind particles (source Krasnopolsky and Mumma 2001). The need for better spectral resolution is evident

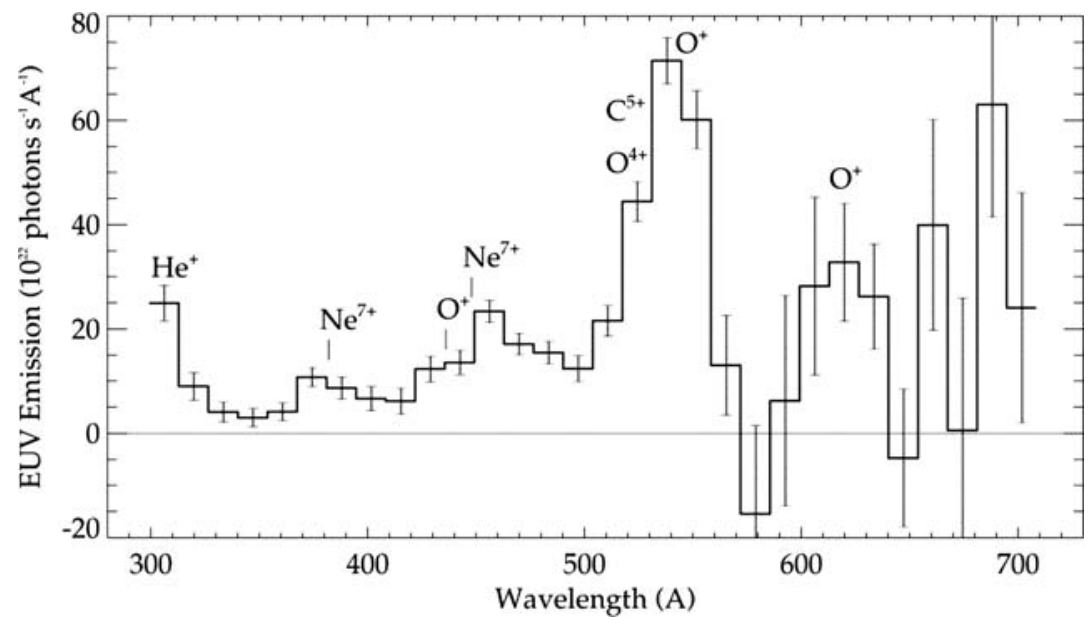

Studies of the atmospheres of Venus and Mars have helped our understanding of the greenhouse effect and the impact of the continuous release of anthropogenic gases such as $\mathrm{CO}_{2}$ into the Earth's atmosphere. The $\mathrm{CO}_{2}$-dominated planets can only be studied in the $\mathrm{UV}$, as telluric $\mathrm{CO}$ and $\mathrm{CO}_{2}$ features severely limit what can be observed from the ground. The cycle of water and water-dissociation products, in particular the deuterated species, allows one to investigate the exchange of water between the surface and the atmosphere and, ultimately, the escape of atmospheric constituents. Recent FUSE observations of the $\mathrm{H}_{2}$ and $\mathrm{D}$ lines showed that in the past Mars was probably covered by a thick water ocean (Yung 1998; Krasnopolsky 2003).

The NO ultraviolet night airglow on Venus was discovered in 1978 by the Pioneer-Venus UV spectrometer; it was followed in 1989 by the detection of the UV day glow. The existence of a suitable orbital platform could have allowed synoptic observations of these phenomena. On 24 June 1999 the Cassini spacecraft flew by Venus. Prominent features detected by the UVIS instrument include the HI $121.6 \mathrm{~nm}$ $(\mathrm{Ly} \alpha)$ line, the OI 130.4 and $135.6 \mathrm{~nm}$, the CI 156.1 and 165.7 nm multiplets, and the CO Fourth Positive bands. Weaker features of NI, CI, CII, OI and CO were also present. The EUV spectrum contained well-defined features at $58.4 \mathrm{~nm}(\mathrm{HeI})$, $83.3 \mathrm{~nm}(\mathrm{OII}), 98.9 \mathrm{~nm}(\mathrm{OI})$, and $102.6 \mathrm{~nm}(\mathrm{HI} \mathrm{Ly} \beta)$. Weaker emissions included OI $104.1 \mathrm{~nm}, \mathrm{CO}(\mathrm{C}-\mathrm{X}) 108.8 \mathrm{~nm}$, NI $113.5 \mathrm{~nm}$, and a blend of CO (B-X) and OI near $115 \mathrm{~nm}$.

The compositon of the upper atmosphere of the giant planets indicates how these planets and their atmospheres originated and evolved. Studies of giant planets in our Solar System provide the knowledge base against which such observations must be interpreted. As an example, let us mention the spectacular Ly $\alpha$ absorption produced by the exoplanet HD209458b during its transits in front of its star disc (Vidal-Madjar et al. 2003).

Images of Jupiter and Saturn auroral emissions with the constantly improving sensitivity of the HST cameras have opened a new era in the understanding of the interaction of giant planets with their magnetic environment. They have revealed the general morphology of the precipitation patterns and their dynamical behaviour. Our knowledge of the complex interaction between the solar wind, the giant planets' magnetospheres, the current systems flowing through these magnetised environments and their signature in the planet ionospheres has thus dramatically improved. In particular, Jupiter's main auroral oval probably results from breakdown of outward corotating and drifting plasma from Io, which drives currents between Jupiter's magnetosphere and ionosphere (Bunce and Cowley, 2001; Grodent et al., 2003). In the region of upward current (downward-moving electrons), field-aligned potentials accelerate electrons to auroral energies, producing the main auroral oval emissions.

Based on HST FUV images, a similar concept has recently been proposed in the case of Saturn. It is now believed that the auroral oval at Saturn corresponds to a ring of upward current bounding the region of open and closed field lines. Following extensive study of Earth's aurora, we now have three cases of magnetosphere-ionosphere coupling whose most dramatic aspect is the aurora. The discovery of the FUV magnetic footprints of Io, Ganymede and Europa, and of Io's trailing tail (Clarke et al., 2002) generated new theories on the acceleration of electrons in the flux tubes and their time stability. Spectral observations of the FUV-EUV $\mathrm{H}_{2}$ line intensity distribution have indicated that the global thermal structure of the giant planet upper atmospheres is strongly controlled by the redistribution of the energy flux occurring after the precipitation of magnetospheric plasma (Grodent et al., 2002).

\section{Cometary physics}

The major result of the early years of exploration in the newly opened UV window was the detection of water dissociation products. The HI Lyman alpha (Ly $\alpha)$ emission was studied 
in detail by Bertaux et al. (1973) and Keller (1973a, 1973b) who revealed the existence of hot hydrogen components in the coma resulting from the photodissociation of water molecules. This naturally led to the proposal by Blamont and Festou (1974) and Keller and Lillie (1974), who measured the scalelength and the production of $\mathrm{OH}$ in comet Kohoutek (1973 XII) and Bennett (1970 II), respectively, that in comets the water molecule was the parent of most observed hydrogen atoms and $\mathrm{OH}$ radicals.

The use of UV and EUV space telescopes allowed the detection of important secondary parent molecule emissions such as those of $\mathrm{CO}, \mathrm{S}_{2}$ and $\mathrm{CS}_{2}$ and the $\mathrm{CO}$ Cameron bands, a proxy for the $\mathrm{CO}_{2}$ molecule. Feldman et al. (2002) used FUSE on C/2000 A2 (LINEAR) to detect new bands of CO at 98.9 and $198.8 \mathrm{~nm}$ and also the $\mathrm{H}_{2}$ emission, as this molecule is a natural breakdown product of water. The overall picture of a comet that emerged around 1980 was that water was indeed the most abundant nuclear species, as first speculated by Whipple (1950).

The measurement of the elemental composition of the UV coma, containing all the most abundant atoms, numerous ions and simple molecules that are dissociation products of nuclear species, yields the elemental composition of the nucleus. As a consequence, as shown by Huebner and Benkhoff (1999), it is possible to investigate the water/CO mixing ratio variation with heliocentric distance. The measurement of the major compound production rates, and their evolution with time, delivers fundamental information on how parent molecules are stored in the comet nuclei and released into the comae upon heating by sunlight.

The observation of species evaporated from the dust in Sun-grazing comets and the discovery of over 700 such comets by SOHO, particularly by the visible-light coronagraphs (e.g., Biesecker et al., 2002), demonstrates the value of observations at low solar elongations. In the UV domain, the results obtained by the SOHO/SWAN instrument (Makinen et al., 2001) show the power of an all-sky Ly $\alpha$ instrument to discover and study comets with low water production rates. The lack of complementary follow-ups, which would have required the use of other instruments capable of low-elongation observations, is obvious. In such cases, spectroscopic observations would likely reveal the presence of many new lines that are usually unseen because of the weakness of the exciting flux.

\section{Asteroids and planetary surfaces}

The reflectivities of planet, asteroid and satellite surfaces are important ingredients in modeling their thermal evolution (solar heating vs. cooling to space), in understanding the weather in these planets, in devising scenarios for the 'chemical' weathering of their surfaces, etc.
Stern et al. (1991) obtained some interesting results regarding the UV reflectivity of Triton and of the binary Pluto/Charon from HST observations, following the optical band surface "mapping" of the two bodies through observations of the mutual events' series in 1985-1990 (Young et al., 2001). Triton's reddish color in the optical does not continue into the UV. Instead, a blue upturn appears shortward of $275 \mathrm{~nm}$. Charon, on the other hand, is grey in the UV as well as in the optical. The differences among these bodies, which are at similar heliocentric distances and probably evolved together, are not currently understood and require more observations.

The UV spectra of Pluto and Charon obtained with the HST (e.g., Krasnopolsky 2001) emphasize the difficulty of obtaining observations of these distant bodies in the UV: these observations demanded 24 and 16 orbits respectively yet yielded only ambiguous results. The availability of an instrument with a larger collecting area, and of a space platform with higher observing efficiency than the HST, is obvious.

\section{The interplanetary medium}

Cosmic dust covers the surfaces of atmosphereless bodies and is an important component that rains down on Earth as visible meteors. In principle, these could originate from the grinding down of TNOs and Main Belt asteroids, or from the disintegration of comets, but the relative ratios are unknown. Each possible source could produce grains with different chemical compositions, porosity, and complexity and this would result in different optical properties that would be exhibited also in the UV.

The grains show evolution under the gravitational influence of the planets, solar radiation pressure, the Yarkovsky and Poynting-Robertson effects, and solar wind drag. Recent observations with the Wisconsin $\mathrm{H} \alpha$ mapper (Reynolds et al., 2004) show that the zodiacal cloud has a definite prograde signature. The broadened widths of the $\mathrm{H} \alpha$ profiles, together with the large amplitude variations in the centroid velocity with elongation angles, indicate that a significant population of the dust grains is in elliptical orbits. These grains could be part of the debris trails of comets seen in IRAS and ISO maps (by Davies et al., 1984; Davies et al., 1997; Sykes et al., 1986; Sykes and Walker, 1992).

The reason to study the Zodiacal Light in detail in the UV is that an accurate measurement of the diffuse UV light in the Solar System is still lacking. Scattering off small dust particles in the zodiacal cloud, at heliocentric distances up to 5-10 a.u., provides the UV light (Leinert et al., 1998). The UV maps of the zodiacal light are much less complete than in other spectral bands. Information about the UV emission from zodiacal dust is necessary for the computation of the thermal equilibrium of dust grains. 
Table 2 Science issues and instrument requirements

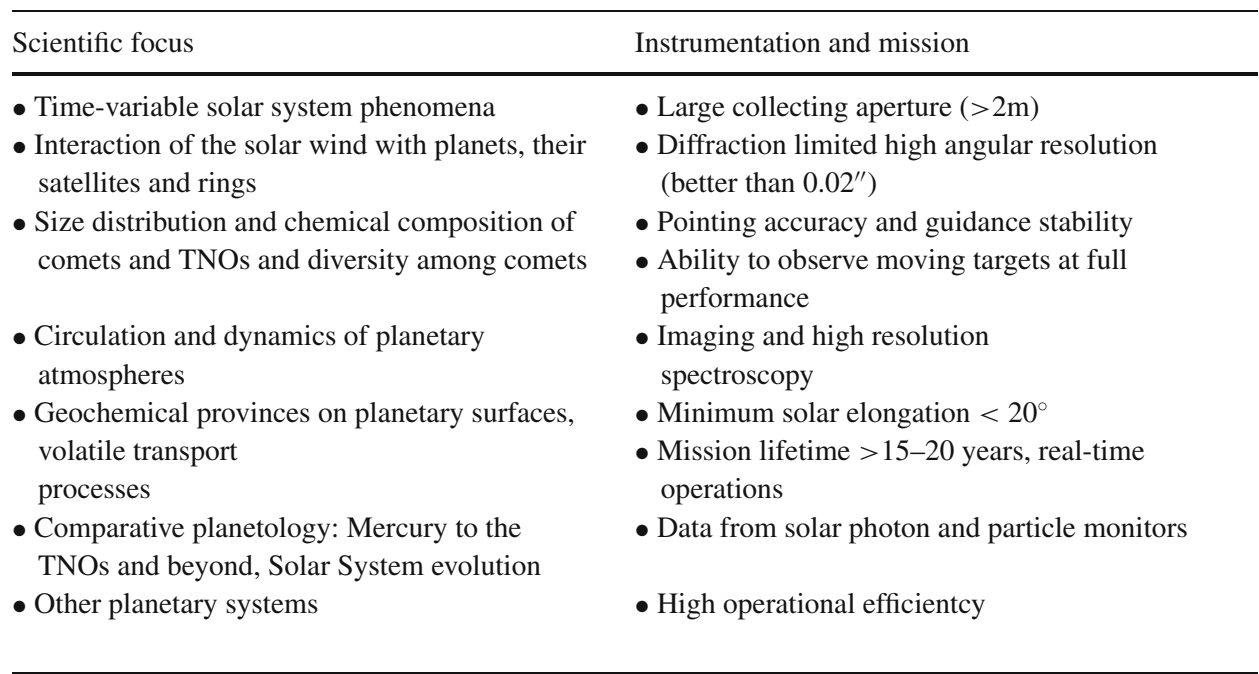

One of the basic parameters in understanding cosmology lies in the measurement of the background light. While this background light component has been well mapped in the IR by COBE/DIRBE, in the optical the still-quoted measurements date back to Pioneer 10 (Toller and Weinberg, 1985). Worse still, in the UV, where the Local Universe is observed, the background emission by the zodiacal light is not well defined at all. The brightness distribution over the sky varies much less than in the optical, where a factor of three between the ecliptic poles and ecliptic equator has been measured. This would imply that while the dust grains that scatter the visible light are somewhat confined to the ecliptic, those that produce the UV zodiacal light are more spherically distributed. The different spatial distribution may reflect a difference in particle size, with the UV scatterers being smaller, on average, than the particles responsible for the optical emission, as well as a possible different origin. Henry and Murthy (1998) also claim the appearance of a spectral feature at $\sim 280$ $\mathrm{nm}$ that has no readily available explanation.

\section{A future UV observatory for solar system studies}

In this section we derive observational requirements for future UV instruments. We start by listing scientific goals that will drive the instrumental requirements. Note that Solar System phenomena are time-variable. The atmospheres of many planets reveal structures and variation with longitude, latitude, and season and everything changes with the solar cycle phase. Such studies require the performance of synoptic observation for all types of objects.

The primary scientific objectives of a UV observatory are a complete characterization of the properties of all types of solar system bodies, from object sizes, internal structure, and rotational properties, to surface properties and atmospheric composition. From these observations, comparisons can be made to determine how solar system objects form and evolve. Table 2 lists a number of science questions in the left column and instrumental requirements in the right column. These requirements are derived from a number of concurrent considerations; for example, large apertures are required not only to compensate for the low solar flux in the UV, but also to counterbalance the low albedos in this spectral region.

Future scientific results

We mentioned above that a particular advantage of observing in the UV is the possibility of achieving a high angular resolution by designing diffraction-limited telescopes used at as short as possible a wavelength. Because of basic optics, the resolving power of the JWST at its main operational band $(4-\mu \mathrm{m})$ can be achieved by a half-meter aperture UV telescope. The WSO/UV telescope, which is one option for a next generation UV telescope facility, will have in imaging mode at $100 \mathrm{~nm}$ a spatial resolution one order of magnitude better than that of the JWST.

Note that the ELT (Extremely Large Telescope) as was discussed in 2005 for possible realization in about two decades, with a filled 100-m aperture, will offer a field of view of three arcmin that, with the adaptive optics option, will have a 1.4 mili-arcsec (mas) resolution with pixels that are less than one mas in size. This angular resolution is now only the province of VLBI, but could be achieved in the UV without the AO option with a $\sim 20$-m spaceborne telescope. In the sub-mm domain, when the Atacama Large Millimetre Array (ALMA) will be operational, its highest angular resolution will be only 5.8 mas (at $720 \mathrm{GHz}$ ).

It is possible to translate this angular resolution into spatial resolution by considering a few examples where we give the linear and angular size of bodies at their typical distances. We ask the reader to consider the amount of information 
retrievable for a nominal angular resolution of one mas ( $\sim$ one $\mathrm{km}$ at one a.u.); note that this is the diffraction limit at 100 $\mathrm{nm}$ for a $20-\mathrm{m}$ telescope while the same limit is $0^{\prime \prime} .01$ for a 2-m telescope.

\section{Planetary atmospheres and magnetospheres}

Some key research areas in the field of atmospheric chemical composition and dynamics are:

- Mars: $\mathrm{CO}_{2}$ and ozone absorptions, transport of water and $\mathrm{CO}_{2}$. Loss of ancient oceans. Dust storms. $\mathrm{CO}_{2}$ recycling.

- The role of cold traps and the transport of condensable species on planets (Mercury, Moon, Mars, Jovian satellites, Triton, Pluto, Charon and TNOs).

- Global circulation of the giant planets, especially the poorly studied planets Uranus and Neptune. The longitudinal distribution of aerosols and UV absorbers can be determined and studied over many successive planet rotations. The comparison of the properties of Uranus ("rolling" along the ecliptic) and of Neptune will be particularly enlightning.

- Study of the evolution of local atmospheric phenomena in giant planets by imaging the different kinds of spots and measuring the evolution of their shape and their transport.

- Distribution of $\mathrm{SO}_{2}$ on Venus and on Io, and its relation to the internal and volcanic activity.

- The solar Ly $\alpha$ line nearly coincides with a line of the fourth positive $(14,0)$ band of $\mathrm{CO}$; fluorescent scattering in the $(14, \mathrm{v})$ band is therefore observable and can be used to monitor this species' abundance in the upper atmosphere of Mars and Venus.

- The emission lines of atomic oxygen at 130.2 and 135.6 $\mathrm{nm}$ in the dayglow spectra of Mars and Venus are very strong and are tracers of the thermospheres of those planets. These observations, coupled with those of $\mathrm{CO}, \mathrm{O}_{3}$ and $\mathrm{CO}_{2}$ (via their absorptions) are essential to understand the aeronomy of the telluric planets, in particular atmospheric species' transport and production/destruction mechanism as a function of solar cycle activity and input of energetic solar wind particles.

- Searches for Ar, $\mathrm{Ne}$ and $\mathrm{N}$ in all atmospheres of solar system objects to constrain the temperature distribution in models of the presolar nebula.

- Use of emissions from $\mathrm{H}, \mathrm{C}, \mathrm{O}, \mathrm{S}$ and $\mathrm{N}$ and their ions to investigate the physics of the planetary ionospheres.

- Investigate on a long term basis the role and sources of neutrals in Saturn's magnetosphere.

- Seach for activity on low-gravity bodies, in particular TNOs, by detecting traces of cryovolcanism through ejection of dust particles.

Sunlight at long UV wavelengths is reflected from stratospheres of planets by a combination of Rayleigh (atomic and molecular) and aerosol scattering. Signature absorptions at specific wavelengths can be used to identify trace organic species (e.g., hydrocarbons, nitriles, etc.), many of which are produced by non-equilibrium processes such as auroral chemistry and photochemistry. A global latitudinal study of the abundance of the atmospheric contituents allows one to separate these two sources of compounds. These tracers can also be used to study the dynamics of the atmospheres. A spatial resolution of order $100 \mathrm{~km}$ at Jupiter would permit unprecendented synoptic observations of the atmosphere of this planet.

The atmosphere-surface interactions at Mars, Io, Ganymede, Mercury, and the Moon could be studied with unprecedented details. The surface of these bodies feeds the atmosphere with volatiles and solid particles and the surface is more or less covered with condensable materials during the planet/satellite seasons. The solid particles play a key role in the thermal balance of some atmospheres (Mars, Titan and possibly Pluto, if the presence of a haze layer in this planet's atmosphere is confirmed).

UV observations allow the characterization and monitoring of the transport of condensable species on planetary surfaces such as those of Mercury, the Moon, Mars, Triton, Io, Pluto and TNOs. The study of volatile escape from lowgravity bodies, in particular TNOs, is greatly facilitated. The transient atmosphere of most low-gravity bodies such as the Moon, Mercury, asteroids, and giant-planet satellites is due to impact of highly energetic particles. The mid-UV region offers the possibility to study most of these atmospheric compounds.

Observations of stellar occultations can be used to obtain atmospheric density profiles. The high time resolution of large instruments allows a very good resolution of the atmosphere height where the occultation takes place. The occultation signature is stronger in the UV because of the higher refraction coefficient. The atmospheric absorptions that could possibly be studied are those of $\mathrm{H}_{2}, \mathrm{CO}_{2}$, and hydrocarbons. Note though that the occulation rate will mostly be determined by the population of occulted stars, which must be rather strong UV emitters, rather than by the spatial resolution of the telescope.

Characterization of the Jovian magnetized environment, and observations of auroral phenomena at Jupiter and Saturn, which is traditionally done with probes in the vicinity of these planets, can be routinely performed with a spaceborne UV telescope. Combining the two options, an orbital spacecraft and a telescope near the Earth as done by e.g., Mauk et al. (2002), would show the similarity of auroral processes on Jupiter and on our Earth. Under this heading we could include also charge-transfer reactions with exospheric gases on low-magnetic field objects (Moon, Mercury, and Mars) and comets. 
Table 3 Physical and Angular size of solar system bodies

\begin{tabular}{llll}
\hline Object & $\mathrm{D}(\mathrm{km})$ & $\mathrm{D}(\operatorname{arcsec})$ & Type of observation \\
\hline Mercury & 4,878 & $4.6-12.5$ & Map of specific regions \\
Venus & 12,102 & $10-60$ & Map of specific regions \\
Moon & 3,476 & 1.865 & Map of specific regions \\
Mars & 6,787 & $14-24$ & Map of specific regions \\
Largest asteroids & Up to 960 & Up to 0.8 & Complete maps \\
Asteroids D $>50 \mathrm{~km}$ & 50 & $>0.05$ & From coarse to detailed maps \\
Jupiter & 143,000 & 47 & Maps of regions of interest \\
Io & 3,640 & 1.2 & Complete map \\
Ganymede & 5,280 & 1.7 & Complete map \\
Saturn + rings & 121,000 & 19.5 & Maps of regions of interest \\
Titan & 5,150 & 0.8 & Complete map \\
Tethys \& Rhea & $1,500-1060$ & $0.17-0.25$ & Complete map \\
Uranus & 52,400 & 4.0 & Complete map \\
Oberon \& Titania & 1,575 & 0.12 & Complete map \\
Neptune & 50,000 & 2.4 & Complete map \\
Triton & 3,200 & 0.15 & Complete map \\
Pluto & 2,200 & 0.1 & Complete map \\
Charon & 1,200 & 0.055 & Coarse map \\
Sedna & 1,600 & 0.037 & Coarse map \\
P/Encke & 5 & 0.007 & A few points \\
P/Halley & 10 & 0.015 & A few points \\
Hale-Bopp & 50 & 0.075 & Coarse map \\
\hline
\end{tabular}

Charged particles moving along magnetic field lines produce auroral phenomena (Jupiter, Saturn). The diagnostic $\mathrm{H}_{2}$ emissions (self-absorbed below $110 \mathrm{~nm}$ ) can be used to monitor this interaction all over the giant planets with special emphasis on auroral regions. The $\mathrm{H}_{2}$ emission is very sensitive to the height of the emissions, and thus may be used to determine the mean energy of the precipitated electrons, as was illustrated with FUSE spectra (Gustin et al., 2004). However, future instruments should have improved spatial resolution so that specific areas of the aurora may be selected.

Equally interesting would be a detailed study of the flux tube footprints in the auroral regions of Jupiter, first identified in HST STIS spectra. The study of the multiple heads observed at the Io magnetic footprint, in particular their relative intensities and inter-separations, and relationship with the location of Io in its plasma torus, requires systematic FUV surveys of Io and its environment at all orbital phases.
Io provides a unique opportunity to study how volcanism is related to the ionosphere of the satellite and to its accompanying torus, while the ensemble is also dependent on the highly dynamic and variable Jovian magnetosphere. The environment of Io and of the Io torus is very hot and many of its emissions (in particular those of the multiply-ionized $\mathrm{O}$, $\mathrm{S}$ and $\mathrm{C}$ ions) are in the EUV and FUV regions. A detailed study of such an intricate system requires synoptic observations over large times scales made at spatial resolutions commensurate with the size of the sources and sinks of the neutral and ionized species. In particular, measurements of FUV emissions from the major ionic species in the torus can provide images of the warm plasma in the main torus region with sufficient spatial resolution to detect detailed structures, yielding a map of plasma conditions dependent on radius, longitude, latitude, and local time. Several unsolved questions concerning the stability and variability of the torus with longitude, local time and intrinsic temporal variability
Fig. 3 The three pictures show the evolution of spatial resolution in observing the Jupiter aurora. The STIS MAMA image (at the right) is the only one showing the trail of the Io magnetic footprint below the auroral oval. Source: Gerard et al. (2003

http://lpap.astro.ulg.ac.be/jupiter)

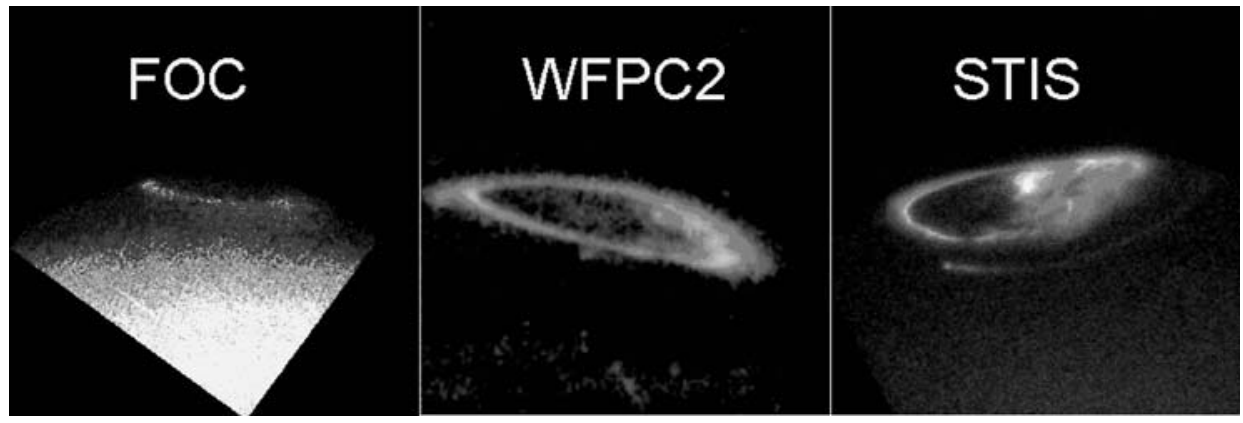


Fig. 4 FUV spectrum of Jupiter's aurora obtained with the FUSE large aperture. The observed intensity distribution of the $\mathrm{H}_{2}$ lines is very well reproduced by the synthetic spectrum for a foreground $\mathrm{H}_{2}$ column of $7 \times 10^{20} \mathrm{~cm}^{-2}$, corresponding to a pressure level of 1 microbar at $800 \mathrm{~K}$ (from Gustin et al., 2004)
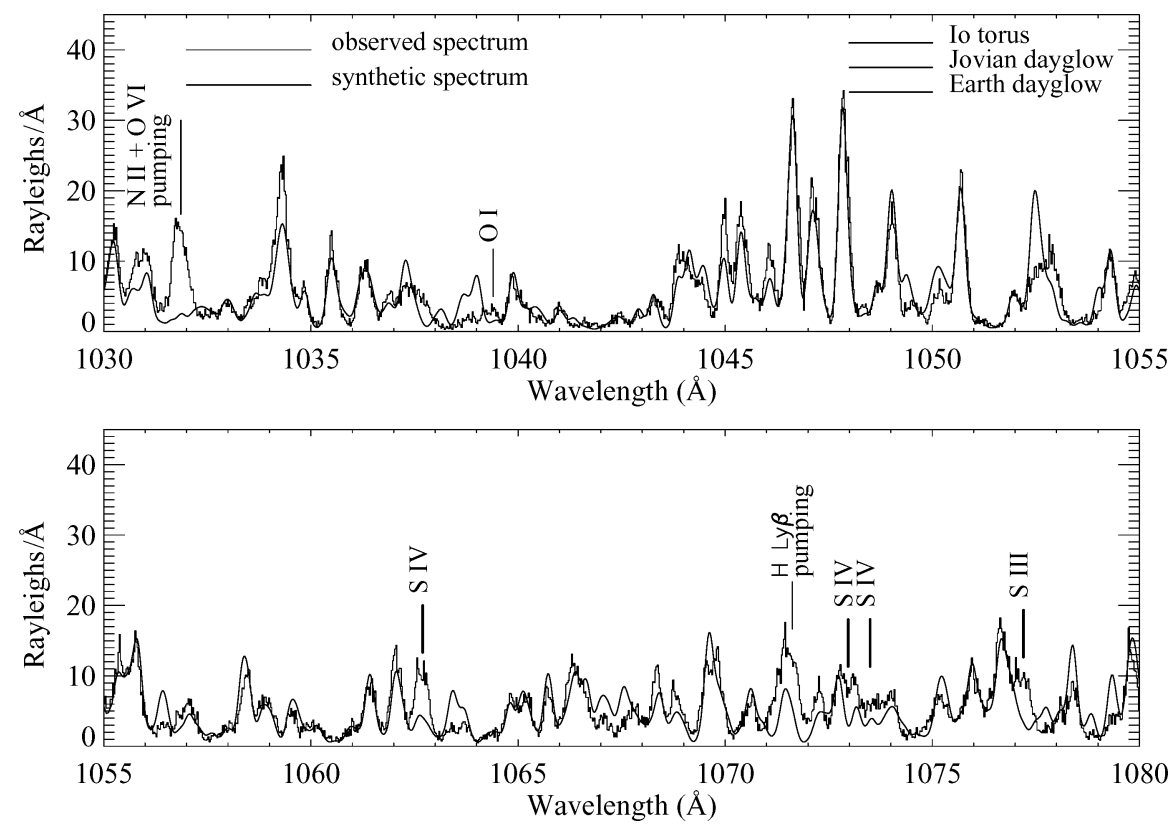

(possibly linked to volcanic activity) can be addressed with multispectral FUV imaging.

While auroral and airglow emissions have been detected by a few UV instruments in the past, none studied in detail the variation of these phenomena as a function of local time, magnetic longitude and solar activity. How these effects control the observed emissions is not yet understood. The magnetospheric activity seems to correlate with the solar activity. A dramatic example of UV imaging capability, emphasizing the need for narrow-band filters and excellent time resolution by using STIS on HST, was given by Waite et al. (2001). The auroral oval light is comprised of the Lyman and Werner bands of molecular hydrogen and the Ly $\alpha$ line of atomic hydrogen. UV observations of the aurora offer a much stronger contrast of the emission against the disk of the planet than does the optical imaging.

It was long assumed that Saturn's magnetosphere and aurora are intermediate between the case of the Earth, where the dominant processes are solar wind driven, and the case of Jupiter, where processes are driven by a large source of internal plasma, the Io torus. Recent HST UV images show instead that Saturn's auroras differ notably from those of the Earth and of Jupiter. Saturn's auroral emissions are only in partial corotation, the auroral oval quickly moves toward higher latitudes in response to solar wind enhancements, and it often exhibits an unexpected "spiral" structure.

The study of the production and evolution of neutrals in the highly variable and neutrals-dominated magnetosphere of Saturn is imperative. Saturn's magnetosphere presents a unique feature: it contains an abnormaly large abundance of neutrals, in particular $\mathrm{H}$ and $\mathrm{OH}$, both dissociation products of water vapour ( $\mathrm{O}$ has recently been detected by the Cassini spectrometer). The source of $\mathrm{OH}$ is poorly known, although it is probably from the sputtering of icy satellite surfaces by energetic ions and interplanetary particles. Saturn will be studied by the UV spectrometer of the Cassini mission, although not on a regular basis because of mission constraints. Paradoxically, one constraint is that the viewing direction is not easily directed towards this large object; remote sensing techniques are actually better suited for such observations

The magnetospheres of Uranus and Neptune are poorly known; the role of their icy and rocky planetary cores on the planet dynamos has not yet been investigated. Why Uranus does not emit as much energy as Neptune is still mysterious. The two planets have very similar interiors but very different rotation axis orientations, hence very different interactions with the solar wind particles. Auroral phenomena are essential to unravel the properties of the magnetic fields of these two distant planets. Neptune and Uranus change their magnetic configuration on time scales shorter than 24 hours because of the relative inclinations of their magnetic and spin axes. Uranus also shows secular changes, because of the high spin-to-orbit inclination. Thus, frequent observations of their auroral activity, achievable only by planetary probes or by a space UV facility, are necessary to investigate these interactions.

Studying the influence of satellites on planetary plasmas and magnetic fields, such as the relation between Io and Jupiter, Triton and Neptune, or between Saturn's satellites and its rings, requires the availability of UV imagers. Although quite spectacular, the results that the UVIS spectrometer on board Cassini (Esposito et al., 2003) will deliver would span only a short time period, in comparison to the time constant of the phenomena to be investigated. Moreover, 
since this instrument resides constantly within the magnetosphere of Saturn, its data would be complementary to what an Earth-based instrument would deliver.

\section{Planetary surfaces and rings}

The repartition of ices and condensable materials due to seasonal effects on Mars, Ganymede, Io, Triton, Pluto, Charon, and the larger TNOs can be studied through albedo maps. The transport of condensable gases on the surface of planetary bodies is controlled by seasonal effects. We are currently witnessing the displacement of the nitrogen frost from the surface of Pluto, and the surface of Triton may possibly be a template for an unchanged Pluto surface since its heliocentric distance hardly varies. In 10-20 years from now, the surface of Pluto should be very different from what it is today and could reveal underlying layers of yet unknown composition. In addition, the UV is an excellent location to look for organic absorptions on these surfaces.

The global characterization and long-term variation of the properties of the rings of Jupiter, Saturn, Uranus and Neptune has not been done (Cassini will do this only during its four-year mission). A space UV facility could monitor the evolution of ring structures coupled to satellite motions by seeing these almost continuously. These observations could determine the lifetime of ring systems and derive the formation times of the existing rings. In low resolution mode, images of Saturn's rings could reveal the amount of absorption by water ice. Note that one may also image the rings in reflection and in absorption at $\mathrm{Ly} \alpha$ to attempt a detection of $\mathrm{H}$, a water dissociation product. As the majority of the absorption features lie below $150 \mathrm{~nm}$, and in order to acquire a significant signal-to-noise result, it would be necessary to observe the ring absorption during occultations of $\mathrm{O}, \mathrm{B}$ and A stars.

The rings can be imaged in the visible or in the NIR (e.g., the $2-\mu \mathrm{m}$ absorption band) but this will not be done at 0.02 " resolution. Also, UV observations will help the search for minor species that are diagnostics of the chemical composition

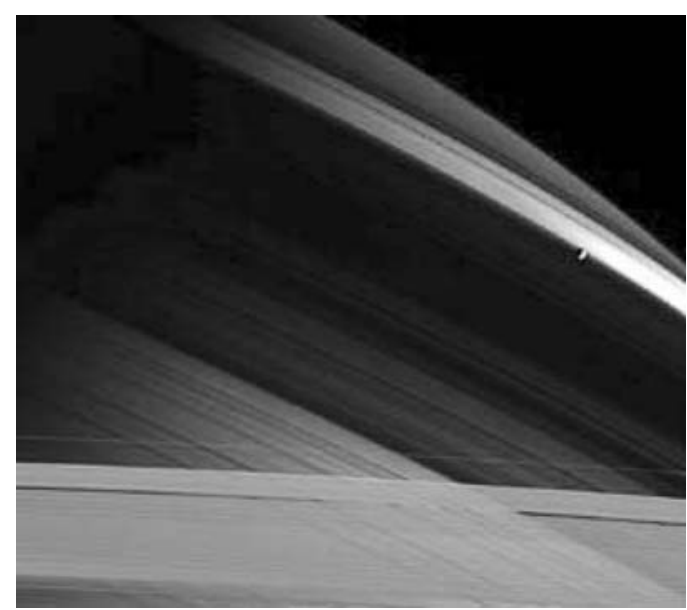

Fig. 6 Saturn's rings imaged by the Cassini probe from 3.7 million $\mathrm{km}$ with a resolution of $22 \mathrm{~km}$ per pixel. Such images could be routinely obtained by a large orbital UV telescope. [Source: JPL PR at http://saturn.jpl.nasa.gov/ cgi-bin/gs2.cgi?path=../multimedia/images/large-moons/images/ PIA06142.jpg\&type $=$ image $]$

of the ring particles. The ring properties can be fairly well represented by assuming that their constituent particles are mostly water ice. However, the red color of the rings is an indication that ice contaminants, probably refractory organics, are present. Since the ring particles are leftovers of the disruption of what was once an icy satellite (or a number of such satellites), a major objective for ring particle studies is the determination of the nature and abundance of these organics. Photometric studies could also shed some light on the abundance of small inorganic particles, as well as on their production and destruction timescales.

\section{Comets}

One of the key goals of cometary studies is to reveal the composition of the solid nucleus, the source of gases and dust particles that scatter sunlight in the coma and tails. Comets have spent most of their life at vast distances from the Sun,
Fig. 5 Polar projections of individual FUV STIS images. The South pole is at the center, noon is downward, dawn to the left and dusk to the right. The left image shows a clear "spiral structure," while the image to the right exhibits a bright spot, tentatively identified as a cusp signature observed at higher latitude, probably due to high-latitude reconnection. (Gérard et al., 2004)
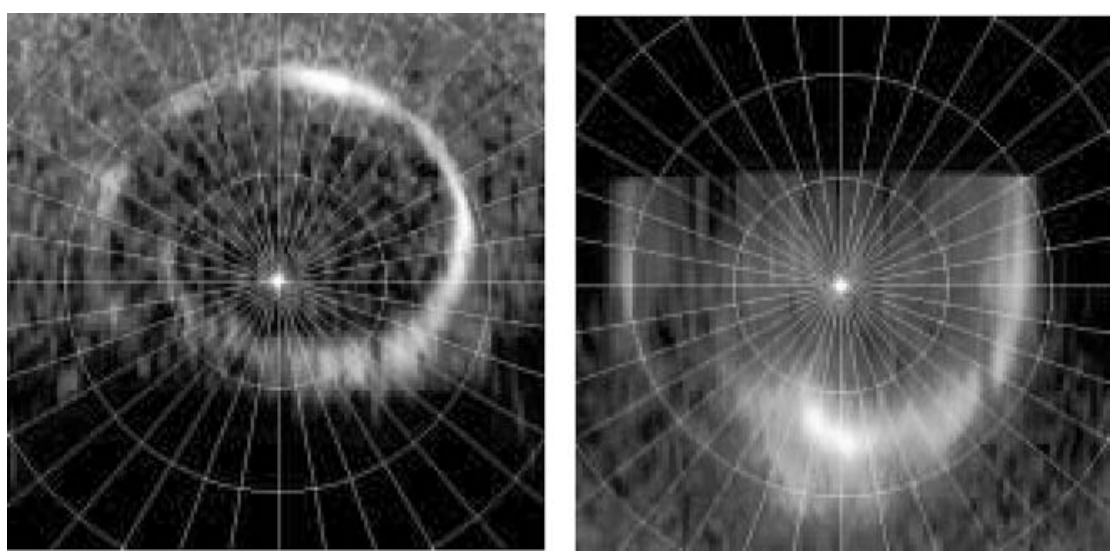
essentially preserved in "deep freeze" in either the TN belt or the Oort cloud until they enter the inner Solar System. If the composition of a cometary nucleus can be determined, then the temperature and density of the material from which these remnants of the primordial solar nebula formed, would be probed. Nuclei cannot be directly studied by telescopic observation except at large heliocentric distances where they are only little shielded or fully unshielded by the gas and dust coma, yet at such distances they are very faint. It is through spectroscopic observations of the coma and tail that key information on the nuclei is obtained. In situ observations concern only a few individual objects and need to be complemented by observations of representatives of the entire population.

Molecules are expelled from the nucleus as this latter is heated by the Sun. The ejected molecules are dissociated by solar photons into molecular fragments and may become ionized. The main nuclear component of comets, water, appears in the coma as atomic $\mathrm{H}$ and $\mathrm{O}$, and as the $\mathrm{OH}$ radical. All three species have very strong transitions in the UV region. The UV is the only domain where the three emissions can simultaneously be detected and imaged at high spatial resolution. Some of the primary molecules, such as $\mathrm{CO}$ and $\mathrm{CO}_{2}$, can also be studied in the UV.

The abundant $\mathrm{CO}_{2}$ molecule can be probed using its ion and dissociation products, all with their strongest transitions in the UV region. The $\mathrm{CO}$ 4th positive bands and the $\mathrm{CO}$ Cameron bands are used for this purpose. The first emission is due to fluorescence of the CO molecule, while the second is produced by prompt emission upon dissociation of the $\mathrm{CO}_{2}$ molecule or by electron impact onto a $\mathrm{CO}$ molecule. UV observations allow one to separate the production mechanisms and distinguish between the emission parent species. Since observations of the strong $\mathrm{CO}_{2}$ band at $4.7 \mu \mathrm{m}$ are difficult, thus rare, observing the $\mathrm{CO}$ Cameron bands is the only way to probe the elusive but cosmogonically important $\mathrm{CO}_{2}$ molecule. The statistical basis for a comparison of the abundances of $\mathrm{CO}$ and $\mathrm{CO}_{2}$ is currently insufficient to derive any firm conclusion, but the existing evidence leans towards a fairly large and stable abundance of the latter molecule in cometary nuclei. Aging processes could be less efficient for $\mathrm{CO}_{2}$ because of the way the molecule is stored in nuclear cometary ices.

Spatial information is crucial to disentangle the various production and excitation mechanisms at play in the coma. The best example is that of $\mathrm{CO}$, which can be produced either directly from the nucleus, from the dissociation of large molecules ejected into the coma, or indirectly by desorption from ejected grains from the surface of the nucleus. The nature of the extended gas sources in the coma needs further investigation, and requires simultaneous UV observations of the $\mathrm{CO}$ and $\mathrm{C} \mathrm{I}$ lines, as well as of the dust grains. High sensitivity observations of the reflectivity of grains near 220 $\mathrm{nm}$ will indicate whether the interstellar absorption bump, observed in the ISM at $217.5 \mathrm{~nm}$, is present in Solar System grains, and if some interstellar grains have survived the collapse of the pre-solar nebula indicating a possible role of these grains as a source of the observed carbon. Radio and NIR observations of CO do not have the sensitivity, or the spatial and temporal resolution of UV observations, leading to coarser results.

The UV region contains emissions of other short-lived nuclear species, namely $\mathrm{S}_{2}$ and $\mathrm{CS}_{2}$ (via the CS emission). These molecules have abundances relative to water that vary greatly from comet to comet, especially $S_{2}$. The storage of these species in the nucleus, and the relation of their outgassing to that of the water and other ices, is unknown. High spatial resolution UV observations are required to reveal their onsets of production and their spatial distributions, hence their production and storage mechanisms. Radio and NIR observations complement the UV in the detection and monitoring of the global production of most parent species, but the spatial resolution achievable in the UV is still unsurpassed in comparison with that in other wavelength domains. The short lifetime of $\mathrm{CS}_{2}$ makes CS an interesting probe for the eventual anisotropic distribution of gas sources on the surface of the nucleus.

The sensitivity of the instrumentation must allow one to completely map the key atomic species $\mathrm{H}, \mathrm{O}, \mathrm{C}, \mathrm{N}$ and $\mathrm{S}$ emissions. Production processes and coma production rates could then be investigated and the global atomic budget of the volatiles in the nucleus obtained. The sensitivity must be sufficient to detect these emissions beyond the Solar System "snow line" at $\sim 5$ a.u. This would allow the detection of yet unseen species, in particular $\mathrm{N}$ or $\mathrm{N}_{2}$ and rare gases. The presence of an $\mathrm{H}$ emission beyond the snow line would directly give the conribution of the non-water $\mathrm{H}$-atom carriers. Since they have low sublimation temperatures, Ar and $\mathrm{Ne}$ with transitions at 104.8 and $106.6 \mathrm{~nm}$ and at $63 \mathrm{~nm}$ respectively can only be trapped in amorphous ice cages, from which they should have escaped rather rapidly if the thermal history of the comets is characterized by high temperature episodes. EUVE and FUSE observations indicate strong depletions of these species by factors of 10-25 but very deep searches still remain to be conducted. Given the fact that $\mathrm{CO}$ has about the same volatility as $\mathrm{Ar}$, one may speculate that the polarity of $\mathrm{CO}$ could explain its high abundance in some comets by providing an electrostatic force to retain it in low-temperature ices.

The EUV region contains numerous ion emissions that allow the detailed study of the solar wind interaction with the comet ionosphere. Observations with FUSE revealed the presence of the important $\mathrm{H}_{2}$ molecule, a direct tracer of water. The Lyman series of atomic hydrogen lines can also be observed. In addition, the $\mathrm{H}_{2}\left(6, v^{\prime \prime}\right)$ bands can be seen because of the coincidence of the $\mathrm{P}_{1}$ line of the $(6,0) \mathrm{H}_{2}$ Lyman system with Ly $\alpha$ (Bowen fluorescence mechanism). 
Fig. 7 IUE UV spectrum of comet Hyakutake

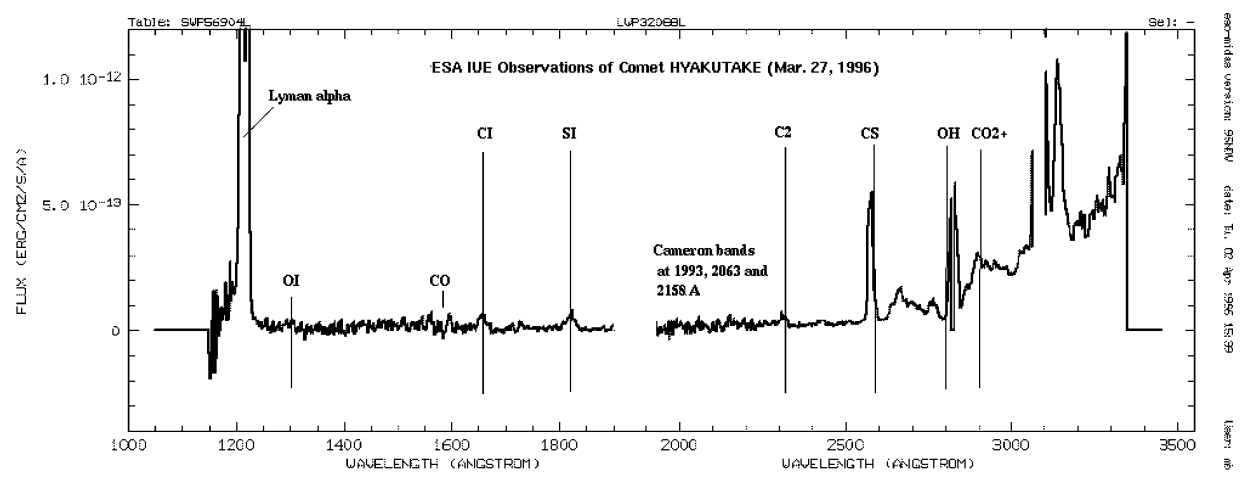

A similar phenomenon explains the strength of the O I 130.2 $\mathrm{nm}$ triplet: the solar $\mathrm{Ly} \beta$ line at $102.572 \mathrm{~nm}$ resonates with the $\mathrm{O} \mathrm{I}{ }^{3} \mathrm{D}-3 \mathrm{P}$ transition at $102.576 \mathrm{~nm}$. The Ne line at $63 \mathrm{~nm}$ could also be detected, thanks to a coincidence with a strong $\mathrm{O} \mathrm{V}$ line.

The FUV region contains the important deuterium line at $121.53 \mathrm{~nm}$, very close to $L y \alpha$, that was first detected in 2004 in comet NEAT (Weaver et al., 2004). Such observations can be used to determine the $\mathrm{D} / \mathrm{H}$ ratio in comets as a function of dynamical type, thus allowing an investigation of the source regions of comets in the solar nebula. Based on our knowledge of the $\mathrm{D} / \mathrm{H}$ ratio in the giant planets, and if comets formed mostly by nebular gas recondensation, one does not expect those formed in the Jupiter region to be as deuterium-rich as those formed at greater heliocentric distances. So far, four comets have yielded a very similar value that could indicate that the $\mathrm{D} / \mathrm{H}$ ratio in comets is mostly fixed by the solid component of the presolar nebula that has probably not been fully evaporated. This hypothesis requires a larger observation sample than presently available to be confirmed.

The interaction of the solar wind with the coma will be studied in detail through:

(1) Observations of the $\mathrm{O}^{+}(83.4 \mathrm{~nm}), \mathrm{N}^{+}(108.5 \mathrm{~nm}), \mathrm{S}^{+}$ $(125.0-125.9 \mathrm{~nm}), \mathrm{C}^{+}(133.5 \mathrm{~nm}), \mathrm{CO}^{+}(200-230$ $\mathrm{nm})$, and $\mathrm{CO}_{2}^{+}(289.0-289.6 \mathrm{~nm})$ emissions,

(2) Emissions produced ahead of the comet by chargeexchange reactions with highly charged solar wind ions. These emissions are spread all over the EUV and FUV regions (some are likely to be present in the FUSE spectra of comets).

The $\mathrm{O}^{+}$and $\mathrm{CO}^{+}$emissions may be the best tracers of the solar wind interaction with the comet. $\mathrm{O}^{+}$will fill out a larger coma volume than $\mathrm{CO}^{+}$and will oppose a weaker force to the solar wind than $\mathrm{CO}^{+}$, a mostly nuclear species with large densities near the nucleus. The slowing down of the solar wind particles will be completely revealed by imaging in these lines. The coma response to a solar wind disturbance will also be documented for the first time, and at high spatial resolution. Note that the $\mathrm{CO}^{+}$lines are hard to separate in the optical from weak $\mathrm{C}_{2}$ and $\mathrm{C}_{3}$ lines, and they appear on top of a sometimes strong continuum. The continuum contribution does not exist, or is extremely weak, in the UV domain and this offers very clean data.

The continuum spectrum of comets below $300 \mathrm{~nm}$ has rarely been studied. The spectrum seems to be solar, with a slope of order $10 \%$ per $100 \mathrm{~nm}$ near $290 \mathrm{~nm}$. Since the albedo of solar system surfaces exibits large changes in the 280-400 nm range, which are caused by the physical as well as the chemical nature of the surface materials, this part of the spectrum should be observed with great care in the hope of measuring a size/chemical nature-dependent change of the albedo curve of the targets with wavelength.

Detailed observations of the UV spectrum of a periodic comet are still to be made. One expects, due to a very different thermal history, that the spectrum of periodic comets would be severely depleted in some highly volatile species, e.g., CO. This can be tested by detecting and monitoring the emissions of $\mathrm{CO}$ and $\mathrm{CO}_{2}^{+}$, or those of $\mathrm{C}$ and $\mathrm{O}$ when the former are not detected.

\section{Other small bodies in the solar system}

Objectives of a UV observational program for small Solar System bodies would be the imaging of the surface, the production of albedo maps, and the characterization of the regolith properties for all objects larger than $50 \mathrm{~km}(0.04 \mathrm{arc} \mathrm{sec}$ at 2 a.u.). The determination of shapes and rotational properties could be inferred, from which the collisional history for these objects would result automatically.

The characterization of the surfaces of asteroids and satellites is traditionally done with optical and NIR information. The UV could add a new dimension to planetary mineralogy, but this would require a baseline laboratory program to characterize the likely minerals in this spectral region. Lunar UV mapping was done for the first time using UIT on the Astro platform (Henry et al., 1995). The Astro results indicate a lunar albedo of $0.038( \pm 10 \%)$ at $170 \mathrm{~nm}$ and no opposition 
effect was found. The reflectivity of the lunar material below $400 \mathrm{~nm}$ was also discussed by Zou et al. (2004).

The requirement for surface characterization is adequate surface resolution: $0.01 \operatorname{arcsec}$ is $25 \mathrm{~km}$ at 3 a.u., necessary to resolve approximately 1,000 Main Belt asteroids or coarsely map TNOs at 60 a.u. with $500 \mathrm{~km}$ resolution. Such angular resolution capabilities, or better, are also necessary in order to resolve binary asteroids to derive their densities, in particular for TNOs. Typical asteroid rotation periods are a few hours; in order to avoid smearing the data, the necessary information must be obtained within $\sim 10$ minutes (surface brightness constraint).

Using the UV to search for comet-like activity in small bodies is beneficial, because in many cases activity manifests itself as grain ejections and these will only scatter sunlight. This diffuse scattered light would be seen in the UV better than in the visible, because of better contrast against the darker sky. The detection of activity from very distant bodies would confirm the predictions of models of thermal evolution at large heliocentric distances, mainly caused by the transport of gases at low temperatures.

The study of transitional objects, dormant and/or extinct comets, is particularly fascinating. The asteroid 3200 Phaeton, the source of the Geminid meteors, is presumably an extinct comet despite the failure to detect a coma (Hsieh and Jewitt, 2005). A claim that asteroid $2001 \mathrm{YB}_{5}$ is the source of a new meteor shower visible on January 7.5 was recently published by Meng et al. (2004). As this object is a Potentially Hazardous Asteroid (PHA), and such objects have been linked theoretically to extinct comets, it is possible that this is another case of a transitional object. Note however a dissenting opinion by Lupishko and Lupishko (2001). The use of UV is beneficial because of the ability to image low surface brightness features; therefore even low levels of activity that would result in a very faint tail or a dusty coma ejection could produce measurable signals.

A search and study of Trojans of all giant planets will help determine whether it is possible that the Trojans were accreted to their present locations at the early stages of solar system formation. Peale (1993) showed that the Lagrangian locations could be stable at the planet accretion phase; the planetesimals could have been captured there by drag in the primeval nebula. The question whether the Trojans are comet-like bodies or not could be solved, as in the case of extinct comets among the NEOs, by searching for comet-like outgasing activity.

A search for multiple asteroid systems in order to determine their density, from which their interior structure could be inferred, can be conducted better with a UV space telescope than with an optical one because of the superior angular resolution that can be achieved. Note that even ESO's Very Large Telescope Interferometer (VLTI) will not achieve a resolution of one mas. With high angular resolution, from a space platform that is not affected by seeing, it would be possible to perform companion searches at much closer distances from the main body of a binary system than if using visible light.

\section{Outer solar system objects}

After the discovery of 90377 Sedna (2003 VB 12 ), it appears that bodies in the inner part of the Oort cloud or scattered out of the TNO belt are observable. Sedna never approaches the Sun closer than $\sim 76$ a.u., it was at about 90 a.u. at discovery, and will become slightly better located for observations in the next decades as it approaches perihelion. This is an opportunity to characterize the nature of a body which has resided in the far outskirts of the Solar System since its formation and can show best the influence of eons of cosmic ray irradiation. Other similar bodies, some possibly larger than Pluto (e.g., 2003 UB $_{313}$ ), exist at tens of a.u. heliocentric distances and should also be included in such studies.

Observations of Sedna and its cousins imply that not only ground-based optical observations from the largest telescopes are required, but also the use of adequate space assets. In particular, the use of low-resolution spectroscopy may be preferable to photometry through a few filters to obtain a global reflectivity profile. Sedna is a $20.5 \mathrm{mag}$ object in the R-band; assuming its UV albedo is only $10 \%$ of its visible one, it would look like a $23^{s t}$ mag UV star. Broadband photometry in the UV, or very low resolution spectroscopy, is possible for such sources using a 2-m class spaceborne telescope. There are tens of other bright TNOs that could be characterized in the FUV region and a new taxonomy for these objects will emerge when all wavelength ranges will have been observed.

Recent models predict cometary activity of TNOs at rather large (tens of a.u.) heliocentric distances. This arises from the influx of heat input at perihelion passage building up the $\mathrm{CO}$ release from within the icy body, combined with transport and recondensation of volatiles within the TNO. The thermal evolution model by Choi et al. (2005) for the scattered TNO $1999 \mathrm{TD}_{10}$ showed an outburst at $\sim 60$ a.u. after 75 orbits! Cometary-like activity has been reported for a number of TNOs, with candidates for activity including the abovementioned scattered TNO (Choi et al., 2003) although this claim was rejected by Mueller et al. (2004) and by Rousselot et al. (2003), as well as the earlier observation of 1996 $\mathrm{TO}_{66}$ (Hainaut et al., 2000). It is possible that cryovolcanism activity could take place, whereby the ejection of a dust/ice grains plume is observed due to pressure build-up in the icy body. Imaging in the UV could facilitate the detection of such cometary activity through the detection of faint dust features because of the low sky background in the UV.

The size distribution of TNOs should be determined to establish to what extent the TN belt is the source of short-period 
comets. Even though faint objects like TNOs will probably not yield a strong signal below $\sim 180-190 \mathrm{~nm}$, and the ice absorption at $175 \mathrm{~nm}$ may not be seen, the advantage in angular resolution of UV observations could allow the estimation of size for some of the objects. The UV segment is necessary also to investigate a possible signature near $220 \mathrm{~nm}$ from possible traces of interstellar compounds that would serve for surface characterization.

\section{Interplanetary material}

A long-term goal could be the complete characterization of the properties of the interplanetary $\mathrm{H}$ and $\mathrm{He}$, and the detection of temporal variations in the emissions/gas temperatures. This, like zodiacal light studies, could be done if a large FOV is available. Apart from a better characterization of the zodiacal light, a large FOV integral field spectrometer would also allow the measurement of the UV spectrum of interplanetary particles and its comparison to that of comet dust and asteroidal surfaces in order to determine the relative contributions of the sources.

The influx of interstellar material into the Solar System may be an elemental ingredient of planetary evolution as the influx of interstellar material could modify the reflection properties of outer Solar System bodies. The Solar System moves through the local ISM at $\sim 20 \mathrm{~km} / \mathrm{sec}$ with the strongest contribution from $\sim 5$ a.u. upstream and with a local cavity extending to $\sim 20$ a.u. downstream. The solar wind interacts with the ISM and reaches equilibrium at 200 a.u., at the heliosphere boundary. Dust particles, detected by the Ulysses spacecraft, have increased by a factor of three from 1997 to 2000. Landgraf et al. (2003) argue that this could increase by another such factor by the end of the next solar cycle, in 2012-3. It is possible to detect very nearby (to the solar system) gas by studying at high resolution the absorption lines of very nearby stars. Frisch (2004) discusses the interaction of the heliosphere with the very nearby ISM. The incoming ISM, detected as gas by Frisch and others, is probably also related to the particulate influx detected by probes in the vicinity of the giant planets.

\section{The Tools}

The proposed Solar System research in the UV relies on several observational techniques. We list these below, before describing the necessary facilities. The techniques, together with the scientific goals of the different projects, set the instrumental constraints of any proposed space facility.
Photometric imaging

This technique yields the position, location and structure of sources. We assume that any instrument designed for imaging will have a photometric capability, good calibration, and long-term stability. In order to be efficient in this task, it should be done in narrow spectral bands centered on strong emisson or absorption features. However, as a database for planetary mineralogy in the ultraviolet range is not yet established, this requires preliminary laboratory work.

Few UV images of solar systems objects not acquired by specific planetary missions exist, mostly obtained during rocket flights or by HST for target acquisition. This is quite surprising, since such data are very powerful and offer a global view of extended objects that helps characterize global variations of the object appearance on a scale of order 1/100 the size of the object and on timescales that vary from minutes to days. Each specific filter requires at least one adjacent "continuum" filter for background subtraction.

Table 4 lists examples of spectral features that could be observed during a program of high-resolution UV imaging. The list is limited to the stronger features shortward of 315 $\mathrm{nm}$, is by no means exhaustive, and no attempt was made to prioritize the spectral bands.

\section{Spectroscopy}

In high resolution mode, $\mathrm{R} \sim 100,000(0.001 \mathrm{~nm}$ resolution at $100 \mathrm{~nm}$ ) is required to resolve plasma velocities in a number of environments such as cometary comae. This has to be achieved at a spatial resolution of 0.05 arcsec or better. In low-resolution mode the emphasis is on detecting and characterizing faint objects, and these figures become 2000 to 3,000 and 0.1 arc sec. An integral field spectrograph is highly desirable as this would reduce the total exposure time. Another solution is a spectrograph like STIS with scanning possibilities.

The efficiency of classical spectrometers is notoriously low, partly, because of the low efficiency of reflection gratings. This has been mitigated by the use of high-efficiency transmission gratings and grisms but it is possible that Volume-Phase Holographic Gratings (VPHG) would offer an additional $50 \%$ gain in efficiency. However, it will be necessary to extend to the UV the present effort, which concentrates on visible and near-IR light.

\section{(Photo)Polarimetry}

Light scattered by dust and magnetized media is often polarized so studies of comet comae and auroral regions could benefit from polarization measurements. For comets, and if the instrument sensitivity is sufficiently high, one could expect to detect the continuum emission down to about $200 \mathrm{~nm}$ 
Table 4 UV Spectral features observable in Solar System targets

\begin{tabular}{|c|c|c|c|}
\hline $\begin{array}{l}\text { Wavelength } \\
(\mathrm{nm})\end{array}$ & Species & $\begin{array}{l}\text { Targets (non- } \\
\text { comprehensive list) }\end{array}$ & Note \\
\hline 58.4 & $\mathrm{HeI}$ & $\begin{array}{l}\text { Interplanetary material, } \\
\text { comets }\end{array}$ & Can be observed in $2^{\text {nd }}$ order \\
\hline 63 & $\mathrm{Ne}$ & Comets, Saturn rings & Resonates with OV \\
\hline 83.4 & OII & Comets, Saturn rings & \\
\hline 98.9 & OI & Comets, Saturn rings & \\
\hline 102.6 & HI Ly $\beta$ & $\begin{array}{l}\text { Interplanetary material, } \\
\text { comets }\end{array}$ & \\
\hline 102.576 & $\mathrm{OI}$ & Comets, Saturn rings & Resonates with $\operatorname{Ly} \beta$ \\
\hline 104.1 & OI & Comets, Saturn rings & \\
\hline 108.5 & NII & Comets & \\
\hline 108.8 & $\mathrm{CO}(\mathrm{C}-\mathrm{X})$ & Comets & \\
\hline $112-115$ & $\begin{array}{l}\mathrm{H}_{2} \text { Lyman \&Werner } \\
\text { bands }\end{array}$ & Giant planets, comets & $\begin{array}{l}\text { Aurora phenomena, satellite } \\
\text { footprints }\end{array}$ \\
\hline 113.5 & NI & Comets, Triton, Pluto & \\
\hline 115 & $\mathrm{CO}(\mathrm{B}-\mathrm{X})+\mathrm{OI}$ & Comets & Blend of features \\
\hline 121.53 & DI Ly $\alpha$ & Comets, IPM, giant planets & \\
\hline 121.6 & HI Ly $\alpha$ & $\begin{array}{l}\text { Interplanetary material, } \\
\text { comets, Mars }\end{array}$ & $\begin{array}{c}\text { The interplanetary } \mathrm{H} \text { I signal can be } \\
\text { absorbed by various } \mathrm{H}_{2} \mathrm{O} \text { clouds }\end{array}$ \\
\hline $125.0-125.9$ & SII & Comets & \\
\hline 130.2 & OI & Comets, Saturn rings & \\
\hline 133.5 & CII & Comets & \\
\hline 135.6 & OI & Comets & \\
\hline 156.1 & $\mathrm{CI}$ & Comets & \\
\hline 165 & $\begin{array}{l}\mathrm{H}_{2} \mathrm{O}^{\text {st }} \text { continuum } \\
\text { band }\end{array}$ & $\begin{array}{l}\text { Comets, Saturn rings, icy } \\
\text { satellites }\end{array}$ & Absorption feature \\
\hline 165.7 & C I & Comets & \\
\hline 175 & Water ice & TNOs & Absorption feature \\
\hline 198.8 & $\mathrm{CO}$ & Comets & \\
\hline $198-220$ & $\mathrm{SO}_{2}$ absorptions & Venus & Surface/atmosphere interaction \\
\hline $200-230$ & $\mathrm{CO}^{+}$ & Comets & \\
\hline 217.5 (wide) & ISM absorption & $\begin{array}{l}\text { Comets, TNOs, zodiacal } \\
\text { dust }\end{array}$ & \\
\hline 250 & Continuum & Comets, zodiacal dust & Dust size distribution \\
\hline 255.4 & $\mathrm{O}_{3}$ Hartley & Mars, Venus, Callisto? & \\
\hline 257.6 & $\mathrm{CS}$ & Comets & \\
\hline $289.0-289.6$ & $\mathrm{CO}_{2}^{+}$ & Comets, Venus, Mars & \\
\hline 300.4 & $\mathrm{O}_{3}$ Hartley & Mars, Venus, Callisto? & \\
\hline 302 & Continuum & Comets & Differential dust size distribution \\
\hline 308 & $\mathrm{OH}$ & Comets, Saturn rings & $\mathrm{H}_{2} \mathrm{O}$ dissociation product \\
\hline
\end{tabular}

and thus collect information on the very small and irregular particles that should polarize these wavelengths more than larger particles.

Asteroid and other atmosphere-less bodies' surfaces will polarize the light they scatter. The polarization properties of asteroid and comets should differ if they are due to particles of different nature and shape. This would also be useful in establishing whether the bodies have been heavily fractured ("rubble piles") or are solid.

\section{Astrometry}

This is the accurate measurement of the position and motion of the source. We assume that any imaging device will have the required electronic and mechanical stability to ensure astrometric capability.

\section{Occultations}

Occultations offer a unique and efficient way of probing the atmosphere of faint bodies such as Pluto, Charon, TNOs, and comets. To be of use the telescope must offer (a) a large collecting area to reach faint targets of occultations, and (b) high observing efficiency to allow the utilization of rare observing opportunities.

Absorptions in comets will mostly be due to water vapour in the first continuum band centered at $165 \mathrm{~nm}$. The IPM Ly $\alpha$ emission could also be absorbed by the water vapour cloud 
surrounding the nucleus once the structure of the H I comet emission has been subtracted. This can be used to map of the water cloud, hence to provide information on its emission pattern at the nucleus.

In spectrographic mode, the absorption of a hot star spectrum must be easily seen in the second continuum absorption of water vapor in bright comets within about $100 \mathrm{~km}$ from the nucleus, if opacities of order $1 / 100$ could be measured. Note that absorptions by coma species other than water are probably much harder to detect, because their column densities times the absorption cross section are significantly smaller. In imaging mode, the opacity of the dust comae can be probed through the observation of O-B-A stars occulted by a comet, from which a column density profile independent of any model assumptions can be derived.

The atmosphere of Pluto, discovered in 1985 by Brosch $(1985,1995)$ when observing a stellar occultation by Pluto and confirmed by other occultations observed from the ground (e.g., Elliot et al., 2003), and the possible existence of an atmosphere around Charon, could be probed during stellar occultations observed in the UV from a space platform. Mink (1993) found 26 possible occultations of stars brighter than $16^{\text {th }}$ mag by Pluto and 25 by Charon from 1993 to 2010 as seen from Earth. The observations of stellar occultations by Pluto during the years following the first atmosphere detection revealed the expansion of the atmosphere while the planet is receeding from perihelion (Elliot et al., 2003). A series of occultation measurements over the years will reveal further secular changes.

The presence of atmospheres around TNOs can be tested for in a similar manner once the number of TNOs with adequate orbits increases and a search is conducted for occultation opportunities. The use of the UV allows the tailoring of the observations to specific absorption bands for definite gases and, for thin atmospheres, allows a higher sensitivity due to the higher refraction index of gases in the UV. On the other hand and as already mentioned above, this requires the identification of an UV-bright star that becomes occulted by the target; the rareness of suitable candidates emphasizes the need for large collecting optics. The large number of TNOs compensates partly for the low occultation rate.

What is currently available?

At present and in the near future UV capabilities are very limited. With the demise of STIS on HST there is now no capability for UV spectroscopy, but some UV imaging is still viable through WFPC 2 and, for small angular extent targets, also with the ACS. Note that UV imaging using CCD detectors is fraught with red leaks of UV filters which prevent the derivation of proper photometry. If an HST refurbishing mission is performed, and if that includes COS, some form of UV spectroscopic capability will return. Similarly, "wide-field" imaging would return with WFC3. Note that long uninterrupted HST observations will always be hampered by the availability of a suitable target in the Continuous Visibility Zone; otherwise typical $30 \mathrm{~min}$ observations are the rule for this and other missions in Low Earth Orbits. With FUSE, the capability exists for FUV spectroscopy from 90 to $119 \mathrm{~nm}$ but for most planetary targets, is very limited by the brightness of the objects.

GALEX offers the capability of low-resolution ( $\sim 7$ arcsec) UV imaging and photometry in two UV bands from 135 to $300 \mathrm{~nm}$, and of low-resolution R 200 "objective prism" mode spectroscopy in the same spectral region. The latter is useful for obtaining the spectral energy distribution in the UV for relatively bright objects, in cases when the objects are at high galactic latitudes (to avoid confusion). Given the use of non-integrating, time-tagged photon detectors in GALEX, this mission has the possibility of electronically "tracking" and following a target for as long as it stays in the field of view, in order to build up the $\mathrm{S} / \mathrm{N}$ or to provide a time-resolved photometric light curve. Similar imaging capabilities, with slightly different collecting apertures, plate scales, and spectral stretch as defined by the filter sets and detector response, will be offered by TAUVEX on GSAT-4 (launch planned for 2006), and by ISRO's ASTROSAT (launch planned for 2007-8), though in both cases the effective photon-collecting efficiency will be similar to that of GALEX. This implies sensitivity sufficient to observe a 12th mag (monochromatic) object in a few seconds with $\mathrm{S} / \mathrm{N} \sim 5$.

UV instruments on planetary probes produce science only when they encounter the planet. Although the information collected by these probes is invaluable, the results are not useful for synoptic studies because of the spotty coverage. The same is true of various small instruments for the Shuttle or the ISS. Similarly, the small imagers on major satellite platforms (e.g., the XMM Optical/UV Monitor) provide a certain very restricted UV capability. The restrictions, in these cases, come from pointing limitations that are dictated by the main satellite instrument, from the low throughput due to the relatively small aperture, and from the tailoring of the filter complement. In most cases, these auxiliary instruments have no spectroscopic capability.

\section{Mission requirements}

In this section we derive the requirements for two future UV telescopes that could satisfy the planetary community's needs until the mid-21st century. The view is guided through the perspective of the HST achievements and aims to provide significant advantages over that mission.

The objectives, which need not be realized by a single mission, would be to: 
- Explore the EUV region simultaneously with the FUV region.

- Operate with a smaller Sun-avoidance angle than HST.

- Have an optical system much faster than the HST, even for a similar size instrument.

- Provide off-axis or Gregorian optics for efficient scattered photon rejection.

- Have solar-blind detectors with high quantum efficiency.

- Have higher observational efficiency (90\% or more of each orbital revolution).

- Avoid contamination by the Earth geocoronal emissions and from the interplanetary H I Ly $\alpha$ background.

- Have near real-time response capability for targets of opportunity.

- Provide higher angular resolution than HST.

- Offer a large number of imaging filters.

- Allow simultaneous use of a number of filters.

- Regain UV capabilities lost with the HST and provide a superior future facility.

- Protect detectors from bright FUV or UV targets.

\section{Spatial resolution}

Aside from the Moon, Venus, Jupiter and Saturn and comets, most Solar System objects have small spatial extensions and require very high spatial resolutions. One hundredth of an $\operatorname{arcsec}$ is required to adequately resolve of order 1,000 asteroids and a few hundred TNOs, or to map planetary surfaces and atmospheric phenomena, and can be achieved with a modest-sized telescope (see table above). A second generation telescope could benefit from the lessons of HST and of the first generation instrument, as well as from techniques developed for telescopes operating in other spectral bands, to yield the milli-arcsec resolution argued for above.

Because of detector limitations, there must be a trade off between spatial resolution and FOV. Large solar system objects such as cometary comae, the zodiacal light, etc., require large FOVs, although key scientific programs need not generally cover the targets entirely. A medium (10 by 10 arcsec at sub-arcsec resolution) and a high-resolution mode ( $1-2$ by $1-2$ arcsec at 0.01 arcsec resolution) should be available.

Apart from the planetary probes, the Earth orbiters are also generally limited by their ability to point accurately at details of the planetary surfaces (or atmospheres) and by the capability for tracking features on planets and satellite. To track the Great Red Spot of Jupiter and keep it (or part of it) in the spectrometer slit, for example, requires a tracking accuracy of $\sim 1 \mathrm{mas} / \mathrm{sec}(1 \mathrm{~km} / \mathrm{sec}$ at 5 a.u., assuming zonal winds of $\sim$ one $\mathrm{km} / \mathrm{sec})$. Any jitter in the tracking would smear the spectral resolution, which will dilute the feature response by that of its neighborhood, or will require closing the tracking loop using imaging in the telescope itself (this task is deemed to be very difficult).

\section{Spectral resolution}

This refers to the capabilities of an instrument operating with any of the two telescopes considered here. The spectral resolution must be matched to the detector's capabilities and to the spectral stretch considered, unless one wishes to consider an echelle configuration.

\section{Pointing range, accuracy and stability}

Targets near the Sun must be observable which imposes a requirement for strong rejection of sunlight. Mercury and comets close to perihelion must be accessible. The minimum elongation angle is of order $15^{\circ}$. If technically not feasible, the elongation limit would be set by Venus (about $40^{\circ}$ ) and by low-q comets, in which case the limit must be as low as possible since comets inside the orbit of Venus are usually extremely bright (this requires protection against damage from bright targets).

Moving target capabilities should allow observations of targets at a rate of at least one arcsec per second of time. Note that the reflex motion of a body at the distance of Neptune is $\sim$ four arcsec/hour.

\section{Spacecraft and spacecraft orbit}

A high apogee orbit is required to allow nearly continuous operations and minimum obscuration by the Earth disc. To avoid penetrating for too long deep into the Earth geocorona, a highly elliptical or at least a geosynchronous orbit is preferrable. It should be possible to use the Earth's shadow to perform observations that require special protection from sunlight. The second Lagrangian point $\mathrm{L}_{2}$ location would be a bonus, but solar panel constraints would restrict the availability of any Solar System target, unless fully orientable solar panels and good stray light suppression are implemented. The radiation background at $\mathrm{L}_{2}$ would be similar to that encountered in any high Earth orbit.

The above-mentioned requirements cannot be realized in a single payload. To achieve an angular resolution of one mas that would be diffraction limited at $100 \mathrm{~nm}$ requires a $20-\mathrm{m}$ aperture. It makes sense to combine this with other cutting-edge technologies that are in design stages now but are likely to mature within one or two decades. Such technologies might include in-space coating of the mirrors with aluminum for best-possible throughput. Such a mission is our long term objective. However, to achieve the goals of quick realization and continuity of UV astronomical efforts, we must also have a mission that would provide a limited 
Table 5 UV missions for Solar System exploration

\begin{tabular}{lll}
\hline Property & Two-meter aperture & Twenty-meter aperture \\
\hline Straylight rejection & $10^{-11}$ & $10^{-13}$ \\
Minimal elongation for observation & $60^{\circ}$ & $20^{\circ}$ \\
Angular resolution@ $100 \mathrm{~nm}$ & $10 \mathrm{mas}$ & $1 \mathrm{mas}$ \\
$5 \sigma$ imaging detection@ $100 \mathrm{~nm}$ & $10^{-16}$ & $10^{-18}$ \\
$\quad$ in one sec [ergs ${ }^{-1} \mathrm{~cm}^{-2} \mathrm{~A}^{-1}$ ] & & \\
Orbit & Geosynchronous or $\mathrm{L}_{2}$ & $\mathrm{~L}_{2}$ or deep space \\
Number of imaging filters & $5 ?$ & $50 ?$ \\
FOV for imaging & Few arcsec & Few arcsec \\
Spectrometer & Single-object, R $\sim 50000$ & Single-object, $\mathrm{R} \sim 200000$ \\
& Long slit, R $\sim 1000$ & Imaging spectrometer, \\
& & $\mathrm{R} \sim 1000$ \\
Stability & $0^{\prime \prime} .1 / \mathrm{sec}$ & $1 \mathrm{mas} / \mathrm{sec}$ \\
Moving target capability & $1^{\prime \prime} / \mathrm{sec}$ & $1^{\prime \prime} / \mathrm{sec}$
\end{tabular}

enhancement with respect to the HST capabilities in the very near future. A longer term goal might include a larger instrument with enhanced capabilitites. Therefore, we conclude with a proposal for two UV space observatories, as detailed in Table 5.

\section{A solar monitor}

Phenomena observed in Solar System objects are either triggered by sunlight and impinging solar particles (resonance fluorescence, excitation of line emissions) or are the consequence of the input of solar energy on them (thermal spectrum, evaporation or sublimation processes). In order to properly interpret the observations of various Solar System bodies, one requires a good knowledge of the solar flux impinging onto these bodies.

The solar spectrum produced in the EUV/FUV regions is due to physical phenomena occuring mosty in the chromosphere and corona of the sun, while the photospheric part dominates beyond $200 \mathrm{~nm}$. Below $150 \mathrm{~nm}$, the solar spectrum is mostly composed of individual lines. The variablity of the solar flux is wavelength-dependent and variabilities of order $10 \%$ are observed in the $150-200 \mathrm{~nm}$ regions and may reach $50 \%$ or so near $120 \mathrm{~nm}$. In the EUV region, flux variations can reach one order of magnitude. These line flux variations translate into highly variable excitation rates of comet transitions on timescales of hours to years. The 27-day and 11 -year cycles are particularly important. The knowledge of the solar spectrum at a resolution of order $0.1 \mathrm{~nm}$ is required to correctly interpret observations of Solar System objects. Because of the slow rotation of the Sun about its axis and, often, significant differences in the ecliptic longitudes of the Earth and the target, observations from the Earth are often not sufficient. Latitudinal solar flux variations and solar line shapes have to be modelled, as they are not easily measurable without complex instrumentation.
In principle, it would be possible to achieve this by observing with a low-resolution spectrometer a point-like constant object that reflects solar radiation, but the requirements for a long duration mission, excellent instrumental stability, and high sampling frequency would make this approach extremely difficult. It is possible to design such an instrument around a small-aperture payload locked onto the Sun. The Solar Radiation Monitor will be a basic tool for planetological research. Because of possible azimuthal differences in the solar emission that may influence the results obtained for other planets and bodies in the system, it is necessary to deploy 2-3 such instruments in orbit around the Sun. As most Solar System objects are confined close to the ecliptic, it is probably not necessary for monitoring purposes to have such instruments in solar polar orbits.

\section{Conclusion}

We argued above the necessity of a two-stage approach in assuring the continued access to the space ultraviolet for the planetary science community. A first stage should be an instrument that would provide the community with better UV capabilities than HST but would be fully dedicated to observations in this spectral segment. Because of this conservative approach, we estimate that this goal could be achieved relatively cheaply and could be implemented almost immediately. If this first step were to be adopted, a suitable instrument could be functioning within five years.

A second stage must represent a breakthrough in all the characteristics of a space telescope and should provide a UV capability commensurate in angular resolution with that of the cutting-edge instruments of the mid21st century: ELT and ALMA. By adopting a 20-m aperture, the throughput with respect to the HST or with the first generation instrument would be increased by two or- 
ders of magnitude. With proper optical construction and platform design, such an instrument could observe Mercury in the ultraviolet and follow Sun-grazing comets relatively soon after their perihelion passage. The angular resolution and sensitivity limit will allow the mapping of Sedna and of other inner Oort cloud objects when these are discovered.

We identified the need for the construction and deployment of solar monitors, to establish the baseline signal that activates atmospheric phenomena in planets, satellites, and comets. We also identified the need for laboratory mineralogic studies to provide the baseline information for UV planetology.

Acknowledgements NB is grateful for continued support of the UV astronomy efforts from the Ministry of Science and Technology of the Israel Government, the Israel Space Agency, and the Austrian Friends of Tel Aviv University. JCG is supported by the Belgian Fund for Scientific Research (FNRS).

NUVA is supported by OPTICON, a project funded by the European Commission under contract RII3-CT-2004-001566

\section{References}

A'Hearn, M.: Icarus 118, 223 (1995)

Bertaux, J.L., Blamont, J.E., Festou, M.: A\&A 25, 415 (1973)

Biesecker, D.A., Lamy, P., St. Cyr, O.C., Llebaria, A., Howard, R.A.: Icarus 157, 323 (2002)

Blamont, J.E., Festou, M.: Icarus 23, 538 (1974)

Brosch, N.: IAUC 4117 (1985)

Brosch, N.: MNRAS 276, 571 (1995)

Bunce, E.J., Cowley, S.W.H.: P\&SS 49, 261 (2001)

Choi, Y.-J., Brosch, N., Prialnik, D.: Icarus 165, 101 (2003)

Choi, Y.-J., Prialnik, D., Brosch, N.: DPS 37, 56.04 (2005)

Clarke, J.T. et al.: Nature 415, 997 (2003)

Davies, J.K. et al.: Nature 309, 315 (1984)

Davies, J.K. et al.: Icarus 127, 251 (1997)

Elliot, J.L. et al.: Nature 424, 165 (2003)

Esposito, L. et al.: Space Science Reviews, in press (2004)

Feldman, P.D., Weaver, H.A., Burgh, E.B.: ApJ 576, 91 (2002)

Frisch, P.C. Adv. Space Res. 34(1), 20 (2004)
Gérard, J.-C. et al.: (http://lpap.astro.ulg.ac.be/jupiter) (2003)

Gérard, J.-C. et al.: J. Geophys. Res. 109(A9), 207 (2004)

Grodent, D. et al.: J. Geophys. Res. 106(A7) (2001)

Grodent, D. et al.: J. Geophys. Res. 108(A7), 1366 (2003)

Gustin, J. et al.: Icarus 171, 330 (2004)

Hahn, J.M. et al.: Icarus 158, 360 (2002)

Hainaut, O.R. et al.: A\&A 356, 1076 (2000)

Henry, R., Murthy, J.: AFRL report from MSX (1998)

Henry, R. et al.: ApJL 454, L69 (1995)

Huebner, W.F., Benkhoff, J.: Space Sci. Rev. 90, 117 (1999)

Hsieh, H., Jewitt, D.: ApJ 624, 1093 (2005)

Keller, H.U.: A\&A 23, 269 (1973a)

Keller, H.U.: A\&A 27, 51 (1973b)

Keller, H.U., Lillie, C.F.: A\&A 34, 187 (1974)

Krasnopolsky, V.A.: Icarus 153, 277 (2001)

Krasnopolsky, V.A., Feldman, W.C.: Science 294, 1914 (2001)

Krasnopolsky, V.A., Mumma, M.J.: ApJ 549, 629 (2001)

Krasnopolsky, V.A. et al.: Science 280, 1576 (1998)

Krasnopolsky, V.A.: Sixth International Conference on Mars (2003)

Landgraf, M. et al.: J. Geophys. Res. 108(A10), LIS5 (2003)

Leinert, Ch. et al.: A\&AS 127, 1 (1998)

Lupishko, D.F., Lupishko, T.A.: Solar System Res. 35, 227 (2001)

Makinen, J.T.T. et al.: A\&A 368, 292 (2001)

Mauk, B.H. et al.: Nature 415, 1003 (2002)

Meng, H. et al.: Icarus 169, 385 (2004)

Mink, D.J.: At the Pluto/Charon conference in Flagstaff (1993)

Mueller, B.T.A. et al.: Icarus 171, 506 (2004)

O'Connell, R.C.: AJ 94, 876 (1987)

Peale, S.J.: Icarus 106, 308 (1993)

Reynolds, R.O., Madsen, G.J., Moseley, S.H. et al.: ApJ 612, 1206 (2004)

Rousselot, P. et al.: A\&A 407, 1139 (2003)

Stern, A. et al.: Icarus 92, 332 (1991)

Sykes, M.V. et al.: Science 232, 115 (1986)

Sykes, M.V., Walker, R.G.: Icarus 95, 180 (1992)

Toller, G.N., Weinberg, J.L.: In: Giese and Lamy (eds.), Properties and Interactions of Interplanetary Dust, Reidel, p. 21 (1985)

Vidal-Madjar, A. et al.: Nature 422, 143; (2003); ApJ 604, L69 (2004)

Young, E.F. et al.: AJ 121, 552 (2001)

Yung, C.: Science 280, 1545 (1998)

Zou, Y.-L. et al.: Chin. J. Astron. Astrophys. 4(1), 97 (2004)

Waite, J.H. et al.: Nature 410, 987 (2001)

Weaver, H.A., A'Hearn, M.F., Arpigny, C., Combi, M.R., Feldman, P.D., Festou, M.C., Tozzi, G.-P.: 36th DPS, paper 23.01, November (2004)

Whipple, F.L.: ApJ 111, 375 (1950) 